SLAC-PUB-9234

hep-th/0205300

\title{
Couplings in Pseudo-Supersymmetry
}

\author{
Matthias Klein同 \\ SLAC, Stanford University, Stanford, CA 94309, USA.
}

\begin{abstract}
We analyze theories in which a supersymmetric sector is coupled to a supersymmetrybreaking sector described by a non-linear realization. We show how to consistently couple $\mathcal{N}=1$ supersymmetric matter to non-supersymmetric matter in such a way that all interactions are invariant under non-linear supersymmetry transformations. We extend this formalism to couple $\mathcal{N}=2$ supersymmetric matter to $\mathcal{N}=1$ superfields that lack $\mathcal{N}=2$ partners but transform in a non-linear representation of the $\mathcal{N}=2$ algebra. In particular, we show how to couple an $\mathcal{N}=2$ vector to $\mathcal{N}=1$ chiral fields in a consistent way. This has important applications to effective field theories describing the interactions of D-brane world-volume fields with bulk fields. We apply our method to study systems where different sectors break different halves of supersymmetry, which appear naturally in models of intersecting branes.
\end{abstract}

May 2002

\footnotetext{
*E-mail: mklein@slac.stanford.edu
} 


\section{Introduction}

With the advent of D-branes [1], string theory has provided new and exciting mechanisms of supersymmetry breaking. Traditionally, most semi-realistic string models were supersymmetric at the string scale and supersymmetry was broken by a field theory effect. The auxiliary field of some supermultiplet would get a vacuum expectation value in the effective supergravity theory. This gave rise to mass splittings for the supermultiplets through the super-Higgs effect. Since the discovery of D-branes, it became clear that there are phenomenologically interesting models where supersymmetry is broken by stringy effects (e.g., [2, 3, [, 5]). Parallel D-branes break half of the supersymmetry that is present in the bulk. The fields arising from the open string excitations, which are confined to the world-volume of the D-branes, only fill multiplets of the smaller supersymmetry algebra. Part of the supersymmetry is explicitly broken on the D-branes, since the world-volume fields lack the corresponding superpartners. Supersymmetry can be completely broken by adding anti-Dbranes, which preserve the other half of the bulk supersymmetry. Thus supersymmetry is broken in a non-local way in such models. Each sector taken separately preserves part of the supersymmetry. A very similar situation arises in models containing stacks of D-branes at angles that intersect each other (e.g., [6, 7]). There is an extended supersymmetry on each stack of D-branes, but only a fraction of this supersymmetry is preserved at each intersection. Supersymmetry is completely broken in models where different intersections break different fractions of supersymmetry.

The field theory of such models is interesting in its own right. Supersymmetry is broken explicitly but non-locally. As a consequence, there are no mass splittings and no quadratic divergences at one-loop [7]. In the present article, we would like to study an effective field theory description of this supersymmetry breaking mechanism.

To determine the couplings of the bulk fields to the boundary fields, it is important to note that the part of supersymmetry that is broken on the D-branes is still non-linearly realized. This statement has not been rigorously proven but there is much evidence in favor of it. For concreteness, consider a single D3-brane in flat ten-dimensional space. From the space-time point of view the translational invariance transverse to the brane is spontaneously broken in the sense that the brane is located at some definite position but all possible positions have degenerate potentials [8]. Similarly, half of the supersymmetries are spontaneously broken. From the world-volume theory point of view the breaking seems to be explicit since the world-volume fields lack the superpartners that would correspond to the broken supersymmetries. However, the broken symmetries are non-linearly realized and it is easy to identify the associated Goldstone fields. In the world-volume theory, position in transverse space is parameterized by the expectation value of the six scalar fields inside the $\mathcal{N}=4$ vector multiplet. These scalars having no potential are the Goldstone bosons of broken translational invariance. Interestingly, the $\mathcal{N}=4$ vector multiplet contains just the right number of fermions to provide four goldstinos corresponding to the breaking of the original

$\mathcal{N}=8$ supersymmetry down to $\mathcal{N}=4$. Let us now compactify four of the transverse dimensions on an orbifold that preserves half of the supersymmetry and place the D3-brane at an orbifold singularity. This projects out half of the fields on the D3-brane world-volume and leads to an $\mathcal{N}=2 U(1)$ gauge theory. The two scalars inside the $\mathcal{N}=2$ vector are 
the Goldstone bosons corresponding to broken translational invariance in the two directions where the brane can still be moved. Again the goldstinos corresponding to the broken supersymmetries are the superpartners of the Goldstone bosons of translation symmetry. If we compactify all six transverse dimensions on an orbifold that preserves only a quarter of bulk supersymmetry and place the D3-brane at an orbifold singularity, then there is no modulus left that would correspond to the motion of the brane in transverse space. Indeed, there is no scalar in the $\mathcal{N}=1$ vector that survives the orbifold projection on the brane.

In a beautiful paper [9], Bagger and Galperin have analyzed the goldstino couplings that are necessary to have non-linearly realized $\mathcal{N}=2$ supersymmetry in a manifestly $\mathcal{N}=1$ invariant $U(1)$ gauge theory. Amazingly, the assumption that the gaugino is the goldstino of partially broken $\mathcal{N}=2$ supersymmetry implies a supersymmetric generalization of the Born-Infeld action for the $U(1)$ vector multiplet. This is further evidence for the conjecture that the supersymmetries that are broken on the D-branes are really non-linearly realized. In this article, we use the formalism of partially broken supersymmetry developed in [9] to determine the bulk-to-boundary couplings, that is the couplings of fields on which $\mathcal{N}=2$ supersymmetry is linearly realized to fields that form non-linear realizations of $\mathcal{N}=2$ supersymmetry. We apply our results to situations where different sectors break different halves of supersymmetry, but all sectors realize supersymmetry (at least) non-linearly. We call this scenario pseudo-supersymmetry.

Additional evidence for non-linearly realized supersymmetry on D-branes was pointed out by the authors of [12] (see also [13]). They argued that consistent gravitino couplings are very constrained and that it is hard to imagine how these constraints can be satisfied without supersymmetry. For a non-supersymmetric type I string model they showed that supersymmetry broken on the D-branes is indeed non-linearly realized. One of the gauginos of the world-volume theory is a gauge singlet and has just the right couplings to be the Goldstone fermion of broken supersymmetry. In contrast to models of spontaneously broken supersymmetry, however, no description of these string models in terms of linear supersymmetry broken by some super-Higgs effect is known. The scale of supersymmetry breaking is not a tunable vacuum expectation value of some field but it is tied to the string scale. The non-linear goldstino couplings have been determined by performing a string computation in 14.

The paper is organized as follows. In the next section we review non-linear realizations of supersymmetry. In section 3, it is shown how to couple $\mathcal{N}=1$ sectors to $\mathcal{N}=0$ sectors in such a way that all interactions are invariant under either linear or non-linear supersymmetry. We then review and generalize the formalism of non-linear realizations applied to partially broken $\mathcal{N}=2$ supersymmetry. This formalism together with the method to couple sectors of non-linear supersymmetry to sectors of linear supersymmetry are used in section 5 to determine the couplings of $\mathcal{N}=2$ multiplets to $\mathcal{N}=1$ matter. In section 6 , we apply these results to a toy model of pseudo-supersymmetry. Some open questions are outlined in the outlook. Finally, our notation is explained in the appendix.

\footnotetext{
${ }^{1}$ This work is related to a problem discussed in [10]. It was motivated by [11] where the couplings of an $\mathcal{N}=2$ bulk vector to $\mathcal{N}=1$ boundary chiral fields were determined. A crucial difference is that in [11], half of the components of the bulk vector were projected out on the boundary, whereas in the present work, all components of bulk fields survive on the boundary.
} 


\section{Review of non-linearly realized supersymmetry}

Supersymmetry is certainly broken at low energies. It is therefore not very surprising that the non-linear realization of supersymmetry was analyzed [15, 16, 17, 18] even before the importance of linear supersymmetry [19] was realized. Let us briefly review the formalism developed in [15, 16, 17] in our notation.

The simplest model of non-linearly realized supersymmetry contains just one fermion, the goldstino $\lambda_{g}$. The supersymmetry variation of the goldstino is [15]

$$
\delta_{\xi} \lambda_{g}=\frac{1}{\kappa} \xi-\kappa v_{\xi}^{m} \partial_{m} \lambda_{g}, \quad \text { where } \quad v_{\xi}^{m}=i \lambda_{g} \sigma^{m} \bar{\xi}-i \xi \sigma^{m} \bar{\lambda}_{g}
$$

The constant $\kappa$ has mass dimension -2 . It can be interpreted as the scale of supersymmetry breaking. We use the spinor conventions of Wess and Bagger [20].

This transformation realizes the supersymmetry algebra [16],

$$
\left[\delta_{\eta}, \delta_{\xi}\right] \lambda_{g}=-2 i\left(\eta \sigma^{m} \bar{\xi}-\xi \sigma^{m} \bar{\eta}\right) \partial_{m} \lambda_{g}
$$

To construct an invariant action, we define [15]

$$
\omega_{m}^{n}=\delta_{m}^{n}-i \kappa^{2} \partial_{m} \lambda_{g} \sigma^{n} \bar{\lambda}_{g}+i \kappa^{2} \lambda_{g} \sigma^{n} \partial_{m} \bar{\lambda}_{g}
$$

and note that

$$
\delta_{\xi} \operatorname{det}(\omega)=-\kappa \partial_{m}\left(v_{\xi}^{m} \operatorname{det}(\omega)\right)
$$

This shows that [15]

$$
\begin{aligned}
S_{\text {goldstino }} & =-\frac{1}{2 \kappa^{2}} \int d^{4} x \operatorname{det}(\omega) \\
& =\int d^{4} x\left(-\frac{1}{2 \kappa^{2}}-\frac{i}{2}\left(\lambda_{g} \sigma^{m} \partial_{m} \bar{\lambda}_{g}-\partial_{m} \lambda_{g} \sigma^{m} \bar{\lambda}_{g}\right)+\mathcal{O}\left(\kappa^{2}\right)\right)
\end{aligned}
$$

is invariant under the non-linear supersymmetry transformation (2.1).

The supersymmetry algebra can be realized on any field $f$ - which may be a Lorentz scalar, spinor, vector or tensor, but we suppress all Lorentz indices for simplicity — by assigning to it the transformation law [16, 17]

$$
\delta_{\xi} f=-\kappa v_{\xi}^{m} \partial_{m} f
$$

It is convenient to introduce covariant derivatives [21],

$$
D_{m} f=\left(\omega^{-1}\right)_{m}^{n} \partial_{n} f
$$

such that $\delta_{\xi}\left(D_{m} f\right)=-\kappa v_{\xi}^{n} \partial_{n}\left(D_{m} f\right)$. To be able to generalize this to gauge covariant derivatives, we assign a transformation law to gauge fields that differs from the standard non-linear realization (2.6) but nevertheless realizes the supersymmetry algebra [22, 23],

$$
\delta_{\xi} A_{m}=-\kappa v_{\xi}^{n} \partial_{n} A_{m}-\kappa\left(\partial_{m} v_{\xi}^{n}\right) A_{n}
$$


This implies that the gauge covariant derivative 23

$$
\mathcal{D}_{m} f=\left(\omega^{-1}\right)_{m}^{n}\left(\partial_{n} f+i A_{n} f\right)
$$

transforms as $\delta_{\xi}\left(\mathcal{D}_{m} f\right)=-\kappa v_{\xi}^{n} \partial_{n}\left(\mathcal{D}_{m} f\right)$ under the non-linear supersymmetry. Finally, we define [23]

$$
\mathcal{F}_{m n}=\left(\omega^{-1}\right)_{m}^{k}\left(\omega^{-1}\right)_{n}^{l}\left(\partial_{k} A_{l}-\partial_{l} A_{k}-i\left[A_{k}, A_{l}\right]\right),
$$

which transforms as $\delta_{\xi} \mathcal{F}_{m n}=-\kappa v_{\xi}^{k} \partial_{k} \mathcal{F}_{m n}$.

It is now clear that

$$
S_{\text {matter }}=\int d^{4} x \operatorname{det}(\omega)\left(-\mathcal{D}_{m} f\left(\mathcal{D}^{m} f\right)^{\dagger}-V(f)-\frac{1}{4} \mathcal{F}^{m n} \mathcal{F}_{m n}\right)
$$

is invariant under (2.1), (2.6), 2.8) for any function $V$. More generally, any Lorentz invariant Lagrangian $\mathcal{L}_{0}$ can be made invariant under the non-linear supersymmetry by coupling it to the goldstino via

$$
\mathcal{L}_{\text {susy }}=\operatorname{det}(\omega) \mathcal{L}^{\prime}=\mathcal{L}^{\prime}+i \kappa^{2}\left(\lambda_{g} \sigma^{m} \partial_{m} \bar{\lambda}_{g}-\partial_{m} \lambda_{g} \sigma^{m} \bar{\lambda}_{g}\right) \mathcal{L}^{\prime}+\mathcal{O}\left(\kappa^{4}\right),
$$

where $\mathcal{L}^{\prime}$ is obtained from $\mathcal{L}_{0}$ by replacing all partial or gauge covariant derivatives by the supercovariant derivatives defined above and $F_{m n}$ by $\mathcal{F}_{m n}$. It is easy to see that $\mathcal{L}_{\text {susy }}$ transforms into a total derivative, $\delta_{\xi} \mathcal{L}_{\text {susy }}=-\kappa \partial_{m}\left(v_{\xi}^{m} \mathcal{L}_{\text {susy }}\right)$.

Sometimes it is useful to consider a different non-linear realization whose action on the goldstino $\tilde{\lambda}_{g}$ is defined by 24, 25]

$$
\delta_{\xi} \tilde{\lambda}_{g}=\frac{1}{\kappa} \xi-2 i \kappa \tilde{\lambda}_{g} \sigma^{m} \bar{\xi} \partial_{m} \tilde{\lambda}_{g}
$$

This transformation is related to the standard transformation through a field redefinition. One can show [21] that $\lambda_{g}\left(x^{m}\right) \equiv \tilde{\lambda}_{g}\left(x^{m}+i \kappa^{2} \lambda_{g} \sigma^{m} \bar{\lambda}_{g}\right)$ transforms as in (2.1). We call (2.13) the chiral version of the non-linear realization (2.1) because it involves only left-handed spinors.

The action of the chiral non-linear supersymmetry on matter fields is given by

$$
\delta_{\xi} f=-2 i \kappa \tilde{\lambda}_{g} \sigma^{m} \bar{\xi} \partial_{m} f
$$

\section{Non-linear realizations from linear realizations}

Consider theories of the form $\mathcal{L}=\mathcal{L}_{1}+\mathcal{L}_{2}+\mathcal{L}_{12}$, where the fields in $\mathcal{L}_{1}$ have no superpartners and realize supersymmetry non-linearly, and the fields in $\mathcal{L}_{2}$ are in $\mathcal{N}=1$ supermultiplets with a linear realization of supersymmetry. The term $\mathcal{L}_{12}$ contains the couplings between the two sectors. We want to find the restrictions on these couplings arising from the requirement of supersymmetry. In other words, we would like to determine how to consistently couple the sector of linearly realized supersymmetry to the sector of non-linearly realized 
supersymmetry. Our strategy will be to find for each superfield $F=\left(f, \psi_{1}, \bar{\psi}_{2}, \ldots\right)$ a composite field $\hat{f}$ consisting of the goldstino and the components of $F$ which, in the limit $\kappa \rightarrow 0$, reduces to $f$, the lowest component of $F$. This composite field has to be chosen such that the non-linear transformation of the goldstino and the linear transformations of the components of $F$ induce the standard non-linear realization on $\hat{f}$. The composite field $\hat{f}$ can be consistently coupled to the sector of broken supersymmetry. Thus, if we know how $f$ couples to the non-supersymmetric matter in the limit $\kappa \rightarrow 0$, then the supersymmetric completion of these couplings is obtained by replacing $f$ by $\hat{f}$.

\subsection{Coupling a chiral superfield to non-supersymmetric matter}

To clarify what we mean by this, let us discuss a simple example. Consider a chiral superfield,

$$
\Phi\left(x_{L}\right)=\phi\left(x_{L}\right)+\sqrt{2} \theta \psi\left(x_{L}\right)+\theta \theta F\left(x_{L}\right), \quad \text { where } x_{L}^{m}=x^{m}+i \theta \sigma^{m} \bar{\theta} .
$$

The supersymmetry transformations are

$$
\begin{aligned}
\delta_{\xi} \phi & =\sqrt{2} \xi \psi, \\
\delta_{\xi} \psi & =i \sqrt{2} \sigma^{m} \bar{\xi} \partial_{m} \phi+\sqrt{2} \xi F, \\
\delta_{\xi} F & =i \sqrt{2} \bar{\xi} \bar{\sigma}^{m} \partial_{m} \psi .
\end{aligned}
$$

It is straightforward to check that the composite field

$$
\hat{\phi}_{L}=\phi-\kappa \sqrt{2} \tilde{\lambda}_{g} \psi+\kappa^{2} \tilde{\lambda}_{g} \tilde{\lambda}_{g} F
$$

transforms precisely according to the chiral non-linear realization (2.14) if the goldstino $\tilde{\lambda}_{g}$ transforms according to $(2.13)$. Moreover, one can verify that

$$
\begin{aligned}
\hat{\phi}\left(x^{m}\right) \equiv & \hat{\phi}_{L}\left(x^{m}+i \kappa^{2} \lambda_{g} \sigma^{m} \bar{\lambda}_{g}\right) \\
= & \phi-\kappa \sqrt{2} \lambda_{g} \psi+\kappa^{2} \lambda_{g} \lambda_{g} F+i \kappa^{2} \lambda_{g} \sigma^{m} \bar{\lambda}_{g} \partial_{m} \phi \\
& +\frac{i}{\sqrt{2}} \kappa^{3} \lambda_{g} \lambda_{g} \partial_{m} \psi \sigma^{m} \bar{\lambda}_{g}+\frac{1}{4} \kappa^{4} \lambda_{g} \lambda_{g} \bar{\lambda}_{g} \bar{\lambda}_{g} \square \phi,
\end{aligned}
$$

where all fields in the last two lines are taken at the argument $x^{m}$, transforms precisely according to the standard non-linear realization (2.6). This shows that the components of the superfield $\Phi$ can be consistently coupled to sectors of non-linearly realized supersymmetry through the combination $\hat{\phi}$.

Let us work out the couplings of a bulk dilaton to brane gauge fields. We consider a model where supersymmetry is completely broken on the brane and the only degrees of freedom in the effective theory are the gauge fields. The bulk is $\mathcal{N}=1$ supersymmetric and the dilaton is in a chiral supermultiplet $\Phi=(\phi, \psi, F)$. Using the above result and the formalism outlined in the previous section, it is easy to find the supersymmetric completion 
of the dilaton coupling term $\left(\phi+\phi^{\dagger}\right) F^{m n} F_{m n}$. By construction,

$$
\begin{aligned}
S & =\int d^{4} x \operatorname{det}(\omega)\left(\hat{\phi}+\hat{\phi}^{\dagger}\right) \mathcal{F}^{m n} \mathcal{F}_{m n} \\
& =\int d^{4} x\left(\left(\phi+\phi^{\dagger}-\kappa \sqrt{2}\left(\lambda_{g} \psi+\bar{\lambda}_{g} \bar{\psi}\right)\right) F^{m n} F_{m n}+\mathcal{O}\left(\kappa^{2}\right)\right)
\end{aligned}
$$

is invariant under the non-linear supersymmetry transformations.

\subsection{General formalism}

It is straightforward to generalize the above result for chiral superfields to arbitrary superfields. We note that (3.4) is just the usual superspace expansion of a chiral superfield with the Grassmann variable $\theta$ replaced by $-\kappa \lambda_{g}$. We will now show that this prescription is valid for any superfield. That is, from the components of an arbitrary superfield $F$, one can build a composite field that transforms as in (2.6) by replacing $\theta \rightarrow-\kappa \lambda_{g}$ in the superspace expansion of $F$. This result was already discovered long ago [21]. We will rederive it here taking a slightly different approach.

Let $F$ be an arbitrary superfield with component expansion

$$
\begin{aligned}
F & =e^{\theta Q+\bar{\theta} \bar{Q}} f \\
& =f+\theta \psi_{1}+\bar{\theta} \bar{\psi}_{2}+\theta \theta m+\bar{\theta} \bar{\theta} n+\theta \sigma^{m} \bar{\theta} v_{m}+\theta \theta \bar{\theta} \bar{\chi}_{1}+\bar{\theta} \bar{\theta} \theta \chi_{2}+\theta \theta \bar{\theta} \bar{\theta} d
\end{aligned}
$$

where the action of the supersymmetry generators $Q, \bar{Q}$ on $f$ is defined by $(\xi Q+\bar{\xi} \bar{Q}) f \equiv \delta_{\xi} f$. The explicit transformation rules for the components of $\mathrm{F}$ are given below.

It is well known that the effect of a supersymmetry transformation acting on $F$ is a shift in superspace [16, 20]

$$
\begin{aligned}
F^{\prime}(x, \theta, \bar{\theta}) & =e^{\xi Q+\bar{\xi} \bar{Q}} F(x, \theta, \bar{\theta}) \\
& =e^{(\theta+\xi) Q+(\bar{\theta}+\bar{\xi}) \bar{Q}+\left(\xi \sigma^{m} \bar{\theta}-\theta \sigma^{m} \bar{\xi}\right) P_{m}} f(x) \\
& =F\left(x^{m}-i\left(\xi \sigma^{m} \bar{\theta}-\theta \sigma^{m} \bar{\xi}\right), \theta+\xi, \bar{\theta}+\bar{\xi}\right)=F\left(x^{\prime}, \theta^{\prime}, \bar{\theta}^{\prime}\right) .
\end{aligned}
$$

The goldstino can be viewed as a hypersurface in superspace defined by

$$
\theta=-\kappa \lambda_{g}(x)
$$

The requirement that this hypersurface be invariant under supersymmetry transformations,

$$
\theta^{\prime}(x)=\theta\left(x^{\prime}\right) \quad \Longrightarrow \quad-\kappa \lambda_{g}^{\prime}(x)+\xi=-\kappa \lambda_{g}\left(x^{\prime}\right),
$$

implies (for infinitesimal $\xi$ )

$$
\lambda_{g}^{\prime}(x)=\lambda_{g}(x)+\frac{1}{\kappa} \xi+i \kappa\left(\xi \sigma^{m} \bar{\lambda}_{g}(x)-\lambda_{g}(x) \sigma^{m} \bar{\xi}\right) \partial_{m} \lambda_{g}(x)
$$


which coincides with the transformation law (2.1). The transformation of a field $\varphi(x)$ in the goldstino background is determined by the condition that $\varphi(x)$ be well-defined on the hypersurface (3.8), i.e., $\varphi^{\prime}(x)=\varphi\left(x^{\prime}\right)$. This implies the transformation law (2.6).

Now, consider the superfield $F$ restricted to the hypersurface (3.8),

$$
\hat{f}(x) \equiv F\left(x,-\kappa \lambda_{g}(x),-\kappa \bar{\lambda}_{g}(x)\right)
$$

Under an infinitesimal supersymmetry transformation it varies as

$$
\delta_{\xi} \hat{f}(x)=i \kappa\left(\xi \sigma^{m} \bar{\lambda}_{g}(x)-\lambda_{g}(x) \sigma^{m} \bar{\xi}\right) \partial_{m} \hat{f}(x)=-\kappa v_{\xi}^{m} \partial_{m} \hat{f}(x)
$$

which is what we wanted to prove. It is straightforward to verify explicitly that

$$
\begin{aligned}
\hat{f}= & f-\kappa \lambda_{g} \psi_{1}-\kappa \bar{\lambda}_{g} \bar{\psi}_{2}+\kappa^{2} \lambda_{g} \lambda_{g} m+\kappa^{2} \bar{\lambda}_{g} \bar{\lambda}_{g} m+\kappa^{2} \lambda_{g} \sigma^{m} \bar{\lambda}_{g} v_{m} \\
& -\kappa^{3} \lambda_{g} \lambda_{g} \bar{\lambda}_{g} \bar{\chi}_{1}-\kappa^{3} \bar{\lambda}_{g} \bar{\lambda}_{g} \lambda_{g} \chi_{2}+\kappa^{4} \lambda_{g} \lambda_{g} \bar{\lambda}_{g} \bar{\lambda}_{g} d
\end{aligned}
$$

varies as in (3.12) using the component field variations

$$
\begin{aligned}
\delta_{\xi} f & =\xi \psi_{1}+\bar{\xi} \bar{\psi}_{2} \\
\delta_{\xi} \psi_{1} & =2 \xi m+\sigma^{m} \bar{\xi}\left(v_{m}+i \partial_{m} f\right) \\
\delta_{\xi} \bar{\psi}_{2} & =2 \bar{\xi}_{n}+\bar{\sigma}^{m} \xi\left(-v_{m}+i \partial_{m} f\right) \\
\delta_{\xi} v_{m} & =\bar{\chi}_{1} \bar{\sigma}_{m} \xi-\frac{i}{2} \xi \sigma_{n} \bar{\sigma}_{m} \partial^{n} \psi_{1}+\bar{\xi}_{m} \bar{\sigma}_{2}+\frac{i}{2} \partial^{n} \bar{\psi}_{2} \bar{\sigma}_{m} \sigma_{n} \bar{\xi} \\
\delta_{\xi} m & =\bar{\xi}_{1}-\frac{i}{2} \partial_{m} \psi_{1} \sigma^{m} \bar{\xi} \\
\delta_{\xi} n & =\xi \chi_{2}+\frac{i}{2} \xi \sigma^{m} \partial_{m} \bar{\psi}_{2} \\
\delta_{\xi} \bar{\chi}_{1}= & 2 \bar{\xi} d+\frac{i}{2} \bar{\sigma}^{n} \sigma^{m} \bar{\xi}_{m} v_{n}+i \bar{\sigma}^{m} \xi \partial_{m} m \\
\delta_{\xi} \chi_{2}= & 2 \xi d-\frac{i}{2} \sigma^{n} \bar{\sigma}^{m} \xi \partial_{m} v_{n}+i \sigma^{m} \bar{\xi} \partial_{m} n \\
\delta_{\xi} d & =\frac{i}{2} \xi \sigma^{m} \partial_{m} \bar{\chi}_{1}-\frac{i}{2} \partial_{m} \chi_{2} \sigma^{m} \bar{\xi}
\end{aligned}
$$

and the goldstino variation (2.1).

It is now clear how to couple the components of the superfield $F$ to bosonic or fermionic fields $\phi_{i}$ without superpartners in such a way that all interactions are invariant under the linear and non-linear supersymmetry transformations acting on $F$ and $\phi_{i}$ respectively. Assume that the coupling of $f$ to the non-supersymmetric fields is of the form $\mathcal{P}\left(f, \partial_{m} f, \phi_{i}, \partial_{m} \phi_{i}\right)$, where $\mathcal{P}$ is some function of the fields and their derivatives. The supersymmetric completion is then obtained by replacing partial derivatives by covariant ones and $f$ by $\hat{f}$. That is, the invariant interaction is $\mathcal{P}\left(\hat{f}, D_{m} \hat{f}, \phi_{i}, D_{m} \phi_{i}\right)$ where $D_{m}$ is defined in (2.7). 
Let us now see how the chiral version of non-linear supersymmetry, eqs. (2.13), (2.14), can be understood in this formalism. A chiral superfield $\Phi$ is a function of $x_{L}$ and $\theta$ only, where

$$
x_{L}^{m}=x^{m}+i \theta \sigma^{m} \bar{\theta} .
$$

In the $\left(x_{L}, \theta\right)$ basis, the superspace expansion of $\Phi$ is simply given by

$$
\Phi=e^{\theta Q} \phi=\phi+\sqrt{2} \theta \psi+\theta \theta F .
$$

A supersymmetry transformation acting on $\Phi$ yields

$$
\begin{aligned}
\Phi^{\prime}\left(x_{L}, \theta\right) & =e^{\xi Q+\bar{\xi} \bar{Q}} \Phi\left(x_{L}, \theta\right) \\
& =e^{(\theta+\xi) Q-\left(2 \theta \sigma^{m} \bar{\xi}+\xi \sigma^{m} \bar{\xi}\right) P_{m}} e^{\bar{\xi} \bar{Q}} \phi\left(x_{L}\right) \\
& =\Phi\left(x_{L}^{m}+2 i \theta \sigma^{m} \bar{\xi}+i \xi \sigma^{m} \bar{\xi}, \theta+\xi\right)=\Phi\left(x_{L}^{\prime}, \theta^{\prime}\right),
\end{aligned}
$$

where we used $\bar{Q}_{\dot{\alpha}} \phi=0$.

The chiral goldstino $\tilde{\lambda}_{g}$ can be viewed as a hypersurface in chiral superspace defined by

$$
\theta=-\kappa \tilde{\lambda}_{g}\left(x_{L}\right)
$$

The requirement that this hypersurface be invariant under supersymmetry transformations,

$$
\theta^{\prime}\left(x_{L}\right)=\theta\left(x_{L}^{\prime}\right) \quad \Longrightarrow \quad-\kappa \tilde{\lambda}_{g}^{\prime}\left(x_{L}\right)+\xi=-\kappa \lambda_{g}\left(x_{L}^{\prime}\right),
$$

implies (for infinitesimal $\xi$ )

$$
\tilde{\lambda}_{g}^{\prime}\left(x_{L}\right)=\tilde{\lambda}_{g}\left(x_{L}\right)+\frac{1}{\kappa} \xi-2 i \kappa \tilde{\lambda}_{g}\left(x_{L}\right) \sigma^{m} \bar{\xi} \partial_{m} \tilde{\lambda}_{g}\left(x_{L}\right)
$$

which coincides with the transformation law (2.13). As a byproduct, we find the relation [25] $\tilde{\lambda}_{g}\left(x_{L}\right)=\lambda_{g}(x)$, where $x_{L}^{m}=x^{m}+i \kappa^{2} \tilde{\lambda}_{g}\left(x_{L}\right) \sigma^{m} \tilde{\lambda}_{g}\left(x_{L}\right)$. The transformation of a field $\varphi\left(x_{L}\right)$ in the chiral goldstino background is determined by the condition that $\varphi\left(x_{L}\right)$ be well-defined on the hypersurface (3.18), i.e., $\varphi^{\prime}\left(x_{L}\right)=\varphi\left(x_{L}^{\prime}\right)$. This implies the transformation law (2.14).

Now, consider the superfield $\Phi$ restricted to the hypersurface (3.18),

$$
\hat{\phi}_{L}\left(x_{L}\right) \equiv \Phi\left(x_{L},-\kappa \tilde{\lambda}_{g}\left(x_{L}\right)\right) .
$$

This is exactly the composite field $\hat{\phi}_{L}$, defined in (3.3). Under an infinitesimal supersymmetry transformation it varies as claimed above,

$$
\delta_{\xi} \hat{\phi}_{L}\left(x_{L}\right)=-2 i \kappa \tilde{\lambda}_{g}\left(x_{L}\right) \sigma^{m} \bar{\xi} \partial_{m} \hat{\phi}_{L}\left(x_{L}\right)
$$

\subsection{Coupling a vector superfield to non-supersymmetric matter}

The case of a vector superfield is slightly more complicated because the supersymmetry algebra only closes up to a gauge transformation. But we can learn some interesting physics by working out the couplings of an $\mathcal{N}=1$ vector to non-supersymmetric matter. 
Consider a vector superfield in the Wess-Zumino gauge

$$
V=-\theta \sigma^{m} \bar{\theta} A_{m}+i \theta \theta \bar{\theta} \bar{\lambda}-i \bar{\theta} \bar{\theta} \theta \lambda+\frac{1}{2} \theta \theta \bar{\theta} \bar{\theta} D .
$$

For simplicity we concentrate on an Abelian vector; the generalization to non-Abelian gauge symmetry is straightforward. The supersymmetry transformations are

$$
\begin{aligned}
\delta_{\xi} A_{m} & =-i \lambda \sigma_{m} \bar{\xi}+i \xi \sigma_{m} \bar{\lambda}, \\
\delta_{\xi} \lambda & =\sigma^{m n} \xi F_{m n}+i \xi D, \\
\delta_{\xi} D & =-\partial_{m} \lambda \sigma^{m} \bar{\xi}-\xi \sigma^{m} \partial_{m} \bar{\lambda} .
\end{aligned}
$$

The couplings of $A_{m}$ to bosonic fields $\phi_{i}$ are of the form

$$
\mathcal{L}_{0}=-\sum_{i}\left(\partial^{m}+i q_{i} A^{m}\right) \phi_{i}\left(\partial_{m}-i q_{i} A_{m}\right) \phi_{i}^{\dagger},
$$

where $q_{i}$ are the charges of the fields $\phi_{i}$. To find the supersymmetric completion of this Lagrangian, we first need to find a superfield whose lowest component is $A_{m}$. This can be easily constructed by computing

$$
\begin{aligned}
\mathcal{A}_{m}= & e^{\theta Q+\bar{\theta} \bar{Q}} A_{m} \\
= & A_{m}+i \theta \sigma_{m} \bar{\lambda}-i \lambda \sigma_{m} \bar{\theta}-\theta \sigma^{n} \bar{\theta}\left(\tilde{F}_{m n}-\eta_{m n} D\right) \\
& -\theta \theta \bar{\theta}\left(\bar{\sigma}_{m n} \partial^{n} \bar{\lambda}-\frac{1}{6} \partial_{m} \bar{\lambda}\right)-\bar{\theta} \bar{\theta} \theta\left(\sigma_{m n} \partial^{n} \lambda-\frac{1}{6} \partial_{m} \lambda\right)+\frac{1}{4} \theta \theta \bar{\theta} \bar{\theta} \partial^{n} F_{m n} .
\end{aligned}
$$

From the previous subsection, we expect that the field that couples covariantly to nonsupersymmetric matter is

$$
\begin{aligned}
\hat{A}_{m}= & A_{m}-i \kappa \lambda_{g} \sigma_{m} \bar{\lambda}+i \kappa \lambda \sigma_{m} \bar{\lambda}_{g}-\kappa^{2} \lambda_{g} \sigma^{n} \bar{\lambda}_{g}\left(\tilde{F}_{m n}-\eta_{m n} D\right) \\
& +\kappa^{3} \lambda_{g} \lambda_{g} \bar{\lambda}_{g}\left(\bar{\sigma}_{m n} \partial^{n} \bar{\lambda}-\frac{1}{6} \partial_{m} \bar{\lambda}\right)+\kappa^{3} \bar{\lambda}_{g} \bar{\lambda}_{g} \lambda_{g}\left(\sigma_{m n} \partial^{n} \lambda-\frac{1}{6} \partial_{m} \lambda\right) \\
& +\kappa^{4} \frac{1}{4} \lambda_{g} \lambda_{g} \bar{\lambda}_{g} \bar{\lambda}_{g} \partial^{n} F_{m n} .
\end{aligned}
$$

The explicit computation of the supersymmetry variation of $\hat{A}_{m}$ shows, however, that $\delta_{\xi} \hat{A}_{m}$ is not exactly of the form (3.12) but rather is given by

$$
\delta_{\xi} \hat{A}_{m}=-\kappa v_{\xi}^{n}\left(\partial_{n} \hat{A}_{m}-\partial_{m} \hat{A}_{n}\right) .
$$

The additional second term is only at first sight unexpected. It can be traced to the fact that the commutator of two supersymmetry transformations acting on $A_{m}$ closes only up to a (field-dependent) gauge transformation,

$$
\left[\delta_{\eta}, \delta_{\xi}\right] A_{m}=2 i\left(\eta \sigma^{n} \bar{\xi}-\xi \sigma^{n} \bar{\eta}\right) F_{m n} .
$$


The term $\kappa v_{\xi}^{n} \partial_{m} \hat{A}_{n}$ in (3.28) is not a gauge transformation, but interestingly, we find that the variation of $\hat{A}_{m}$ agrees with (2.8) up to a gauge transformation.

$$
\delta_{\xi} \hat{A}_{m}=-\kappa v_{\xi}^{n} \partial_{n} \hat{A}_{m}-\kappa \partial_{m} v_{\xi}^{n} \hat{A}_{n}+\kappa \partial_{m}\left(v_{\xi}^{n} \hat{A}_{n}\right) .
$$

This allows us to build gauge covariant derivatives defining

$$
\mathcal{D}_{m} \phi_{i}=\left(\omega^{-1}\right)_{m}^{n}\left(\partial_{n} \phi_{i}+i q_{i} \hat{A}_{n} \phi_{i}\right)
$$

To cancel the gauge variation of $\hat{A}_{m}$ under $\delta_{\xi}$, we need to modify the non-linear supersymmetry transformation of $\phi_{i}$ from the standard form (2.6) to

$$
\delta_{\xi} \phi_{i}=-\kappa v_{\xi}^{m}\left(\partial_{m} \phi_{i}+i q_{i} \hat{A}_{m} \phi_{i}\right)
$$

A short calculation shows that the Lagrangian

$$
\mathcal{L}=-\operatorname{det}(\omega) \sum_{i} \mathcal{D}^{m} \phi_{i}\left(\mathcal{D}_{m} \phi_{i}\right)^{\dagger}
$$

is indeed invariant up to a total derivative under the above supersymmetry transformations.

\section{Partially broken extended supersymmetry}

Let us see how the formalism presented in the preceding sections generalizes to the case of non-linearly realized $\mathcal{N}=2$ supersymmetry. We are interested in situations where an $\mathcal{N}=1$ subgroup is still linearly realized, i.e., the extended supersymmetry is partially broken. Specifically, we focus on cases where the spectrum and all interactions are manifestly $\mathcal{N}=1$ supersymmetric but the $\mathcal{N}=1$ superfields have no $\mathcal{N}=2$ partners. The goldstino $\lambda_{g}$ resides in an $\mathcal{N}=1$ supermultiplet $\Lambda_{g}$ in such models. This can be a chiral [26], a vector [9] or a linear multiplet [27]. We concentrate on the case where the goldstino is the superpartner of a $U(1)$ gauge boson because this seems to be most appropriate for the study of supersymmetry breaking on D-branes.

\subsection{An action for the goldstino superfield}

We start by considering a model that only contains the goldstino and its superpartner, a $U(1)$ gauge boson. The generalization of the goldstino transformation law (2.1) is [28]

$$
\delta_{\eta}^{*} \Lambda_{g}=\frac{1}{\kappa} \eta-\kappa V_{\eta}^{m} \partial_{m} \Lambda_{g}, \quad \text { where } \quad V_{\eta}^{m}=i \Lambda_{g} \sigma^{m} \bar{\eta}-i \eta \sigma^{m} \bar{\Lambda}_{g}
$$

$\Lambda_{g}$ is a superfield with $\lambda_{g}$ as its lowest component and the star on $\delta_{\eta}^{*}$ is to remind us that we are varying with respect to the second supersymmetry. Again, it is useful to also consider a chiral version of this transformation law [28], which generalizes (2.13),

$$
\delta_{\eta}^{*} \tilde{\Lambda}_{g}=\frac{1}{\kappa} \eta-2 i \kappa \tilde{\Lambda}_{g} \sigma^{m} \bar{\eta} \partial_{m} \tilde{\Lambda}_{g}
$$


This transformation is related to the standard transformation (4.1) through the field redefinition $\Lambda_{g}\left(x^{m}, \theta, \bar{\theta}\right) \equiv \tilde{\Lambda}_{g}\left(x^{m}+i \kappa^{2} \Lambda_{g} \sigma^{m} \bar{\Lambda}_{g}, \theta, \bar{\theta}\right)$.

Since the goldstino superfield $\Lambda_{g \alpha}$, where $\alpha$ is a Weyl spinor index, has the same lowest component as the field strength superfield $W_{g \alpha}$ associated to the $U(1)$ gauge boson, it is natural to expect that one could identify these two superfields, $\Lambda_{g \alpha} \sim W_{g \alpha}$. This, however, can only be true to zeroth order in an expansion in powers of $\kappa$. The reason is that the transformation (4.1) is not compatible with the conditions that have to be satisfied by the field strength superfield:

$$
\bar{D}_{\dot{\alpha}} W_{g \beta}=0, \quad D^{\alpha} W_{g \alpha}=\bar{D}_{\dot{\alpha}} \bar{W}_{g}^{\dot{\alpha}}
$$

Note that an identification of the form $\tilde{\Lambda}_{g \alpha} \sim W_{g \alpha}$ is not possible either, because (4.2) is only compatible with the first but not with the second condition in (4.3).

If one insists that the goldstino is the lowest component of $W_{g \alpha}$, one has to find a transformation law for $W_{g \alpha}$ which differs from (4.1) but still realizes the supersymmetry algebra and is compatible with the conditions (4.3). This transformation was determined by the authors of [9] and is given by?

$$
\delta_{\eta}^{*} W_{g \alpha}=\frac{2}{\kappa} \eta_{\alpha}+\frac{\kappa}{2} \bar{D}^{2} \bar{X} \eta_{\alpha}+2 i \kappa\left(\sigma^{m} \bar{\eta}\right)_{\alpha} \partial_{m} X
$$

where $X$ is a chiral superfield (of mass dimension 3), determined through the recursive relation

$$
X=\frac{\frac{1}{4} W_{g}^{2}}{1+\frac{\kappa^{2}}{4} \bar{D}^{2} \bar{X}} .
$$

Using the tricks explained in [9], this relation can be explicitly solved for $X$. In our notation, the result is

$$
\begin{aligned}
& X=\frac{1}{4} W_{g}^{2}-\frac{\kappa^{2}}{32} \bar{D}^{2}\left[\frac{W_{g}^{2} \bar{W}_{g}^{2}}{1-\frac{1}{2} A+\sqrt{1+\frac{1}{4} B^{2}-A}}\right] \\
& \text { where } \quad A=-\frac{\kappa^{2}}{8}\left(D^{2} W_{g}^{2}+\bar{D}^{2} \bar{W}_{g}^{2}\right) \text {, } \\
& B=-\frac{\kappa^{2}}{8}\left(D^{2} W_{g}^{2}-\bar{D}^{2} \bar{W}_{g}^{2}\right) \text {. }
\end{aligned}
$$

To verify that (4.4) realizes the supersymmetry algebra, note that one has [9]

$$
\delta_{\eta}^{*} X=\frac{1}{\kappa} W_{g} \eta
$$

An immediate consequence of the $X$ transformation law (4.7) is that

$$
\begin{aligned}
\mathcal{L} & =\int d^{2} \theta X+\int d^{2} \bar{\theta} \bar{X} \\
& =\frac{1}{4} \int d^{2} \theta W_{g}^{2}+\frac{1}{4} \int d^{2} \bar{\theta} \bar{W}_{g}^{2}+\frac{\kappa^{2}}{8} \int d^{2} \theta d^{2} \bar{\theta} W_{g}^{2} \bar{W}_{g}^{2}+\mathcal{O}\left(\kappa^{4}\right)
\end{aligned}
$$

\footnotetext{
${ }^{2}$ Our notation and conventions are summarized in the appendix. They differ from those of [0.
} 
is invariant up to a total derivative under the second supersymmetry. This reduces to the usual Lagrangian for a supersymmetric gauge multiplet in the limit $\kappa \rightarrow 0$. The exciting feature of the Lagrangian (4.8) is that its restriction to the bosonic terms coincides precisely with the Born-Infeld Lagrangian [29]. Indeed, it has been shown in [9] that the bosonic terms of (4.8) can be written as

$$
\mathcal{L}_{\text {bos }}=\frac{1}{\kappa^{2}}\left(1-\sqrt{-\operatorname{det}\left(\eta_{m n}+\kappa F_{m n}\right)}\right) .
$$

The requirement of non-linearly realized $\mathcal{N}=2$ supersymmetry implies an $\mathcal{N}=1$ supersymmetric generalization of the Born-Infeld action for the goldstino superfield if we assume that the goldstino resides in a vector multiplet.

We are now in a position to give the precise relationship between $W_{g}$ and $\tilde{\Lambda}_{g}$ that transforms according to the chiral standard transformation. One can verify that the superfield

$$
\tilde{\Lambda}_{g \alpha} \equiv \frac{\frac{1}{2} W_{g \alpha}}{1+\frac{\kappa^{2}}{4} \bar{D}^{2} \bar{X}}
$$

transforms as in (4.2). In terms of $\tilde{\Lambda}_{g}$, the Lagrangian (4.8) can be written as

$$
\begin{gathered}
\qquad \mathcal{L}=\int d^{2} \theta E_{L} \tilde{\Lambda}_{g} \tilde{\Lambda}_{g}+\int d^{2} \bar{\theta} E_{R} \overline{\tilde{\Lambda}}_{g} \overline{\tilde{\Lambda}}_{g}, \\
\text { where } \quad E_{L}=1+\frac{\kappa^{2}}{4} \bar{D}^{2} \bar{X}, \quad E_{R}=1+\frac{\kappa^{2}}{4} D^{2} X .
\end{gathered}
$$

Note that $\tilde{\Lambda}_{g}$ and $E_{L}$ are chiral superfields, i.e., $\bar{D}_{\dot{\alpha}} \tilde{\Lambda}_{g \beta}=0=\bar{D}_{\dot{\alpha}} E_{L}$. From (4.7), one finds that $E_{L}$ transforms as a chiral density under the non-linear supersymmetry transformation,

$$
\delta_{\eta}^{\star} E_{L}=-i \kappa \partial_{m} W \sigma^{m} \bar{\eta}=-2 i \kappa \partial_{m}\left(E_{L} \tilde{\Lambda}_{g} \sigma^{m} \bar{\eta}\right)
$$

\section{2 $\mathcal{N}=1$ matter fields in the goldstino background}

With the above results, it is straightforward to include $\mathcal{N}=1$ matter fields that couple to the goldstino in such a way that all interactions are invariant under the non-linear $\mathcal{N}=2$ supersymmetry. A chiral superfield $\tilde{\Phi}$ transforms as 28]

$$
\delta_{\eta}^{\star} \tilde{\Phi}=-2 i \kappa \tilde{\Lambda}_{g} \sigma^{m} \bar{\eta} \partial_{m} \tilde{\Phi}
$$

From the transformation law (4.12) of the chiral density $E_{L}$, we find that

$$
\mathcal{L}_{\text {pot }}=\int d^{2} \theta E_{L} \mathcal{P}(\tilde{\Phi})+\int d^{2} \bar{\theta} E_{R} \mathcal{P}\left(\tilde{\Phi}^{\dagger}\right)
$$

transforms into a total derivative for an arbitrary analytic function $\mathcal{P}$. This generalizes the leading order result obtained in [9] to all orders in $\kappa$. 
To find a generalization of the kinetic terms $\int d^{2} \theta d^{2} \bar{\theta} \Phi^{\dagger} \Phi$, we define

$$
\begin{aligned}
\Lambda_{g}(x, \theta, \bar{\theta}) & =\tilde{\Lambda}_{g}\left(x^{m}+i \kappa^{2} \Lambda_{g} \sigma^{m} \bar{\Lambda}_{g}, \theta, \bar{\theta}\right) \\
\Phi(x, \theta, \bar{\theta}) & =\tilde{\Phi}\left(x^{m}+i \kappa^{2} \Lambda_{g} \sigma^{m} \bar{\Lambda}_{g}, \theta, \bar{\theta}\right) \\
E(x, \theta, \bar{\theta}) & =E_{L}\left(x^{m}+i \kappa^{2} \Lambda_{g} \sigma^{m} \bar{\Lambda}_{g}, \theta, \bar{\theta}\right)
\end{aligned}
$$

and note that $\Lambda_{g}$ transforms as the goldstino superfield (4.1) and $\Phi$ transforms as

$$
\delta_{\eta}^{\star} \Phi=-i \kappa\left(\Lambda_{g} \sigma^{m} \bar{\eta}-\eta \sigma^{m} \bar{\Lambda}_{g}\right) \partial_{m} \Phi=-\kappa V_{\eta}^{m} \partial_{m} \Phi
$$

This is the standard non-linear realization for a general superfield. A natural guess for an invariant action is

$$
\begin{aligned}
& \qquad \mathcal{L}_{\text {kin }}=\int d^{2} \theta d^{2} \bar{\theta} \hat{E} K\left(\Phi, \Phi^{\dagger}\right) \\
& \text { where } \quad \hat{E}=\frac{1}{2}\left(E+E^{\dagger}\right)=1+\frac{\kappa^{2}}{8} \bar{D}^{2} \bar{\Lambda}_{g}^{2}+\frac{\kappa^{2}}{8} D^{2} \Lambda_{g}^{2}+\mathcal{O}\left(\kappa^{4}\right),
\end{aligned}
$$

and the Kähler potential $K\left(\Phi, \Phi^{\dagger}\right)$ is an arbitrary real function of $\Phi, \Phi^{\dagger}$. To order $\kappa^{2}$, this agrees with the Lagrangian of [9]. It is easy to check that the $\mathcal{O}(1 / \kappa)$ and the $\mathcal{O}(\kappa)$ terms cancel in the variation of the Lagrangian. Whether the invariance also holds to higher orders in $\kappa$ is not clear to us.

A vector superfield $V$ can be chosen to transform according to the standard non-linear realization (4.16),

$$
\delta_{\eta}^{\star} V=-\kappa\left(i \Lambda_{g} \sigma^{m} \bar{\eta}-i \eta \sigma^{m} \bar{\Lambda}_{g}\right) \partial_{m} V
$$

The transformation of the field strength superfield $W_{\alpha}$ derived from the vector $V$ is nonstandard and very complicated. But it is possible to construct an $\mathcal{N}=2$ covariant field strength $\mathcal{W}_{\alpha}$ that transforms according to (4.13). This implies that

$$
\mathcal{L}_{\text {gauge }}=\int d^{2} \theta d^{2} \bar{\theta} \hat{E} \Phi^{\dagger} e^{V} \Phi+\frac{1}{4} \int d^{2} \theta E_{L} \mathcal{W} \mathcal{W}+\frac{1}{4} \int d^{2} \bar{\theta} E_{R} \overline{\mathcal{W}} \overline{\mathcal{W}}
$$

is invariant up to a total derivative.

To obtain $\mathcal{W}_{\alpha}$, we have to introduce covariant derivatives $\mathcal{D}_{\alpha}, \overline{\mathcal{D}}_{\dot{\alpha}}, D_{m}$ that reduce to $D_{\alpha}, \bar{D}_{\dot{\alpha}}, \partial_{m}$ in the limit $\kappa \rightarrow 0$ and satisfy

$$
\delta_{\eta}^{\star}\left(\mathcal{D}_{\alpha} V\right)=-\kappa V_{\eta}^{m} \partial_{m}\left(\mathcal{D}_{\alpha} V\right)
$$

and similarly for $\overline{\mathcal{D}}_{\dot{\alpha}}, D_{m}$. The explicit expressions for $\mathcal{D}_{\alpha}, \bar{D}_{\dot{\alpha}}, D_{m}$, as derived by the authors of [9], are given in the appendix. It follows that

$$
\mathcal{W}_{\alpha}^{0}=-\frac{1}{4} \overline{\mathcal{D}}^{2} \mathcal{D}_{\alpha} V
$$

transforms according to the standard non-linear realization (4.16). (For simplicity, we concentrate on an Abelian gauge symmetry.) However, $\mathcal{W}_{\alpha}^{0}$ is not gauge invariant. 
To understand the reason for this, let us first see how gauge invariance is achieved for the $\mathcal{N}=1$ field strength superfield $W_{\alpha}=-\frac{1}{4} \bar{D}^{2} D_{\alpha} V$. A gauge transformation acts as $V \rightarrow V+i\left(\Lambda-\Lambda^{\dagger}\right)$ on the vector superfield, where $\bar{D}_{\dot{\alpha}} \Lambda=0$. The latter condition ensures that a chiral superfield $\Phi$ remains chiral under a gauge transformation $\Phi \rightarrow e^{-i \Lambda} \Phi$. Gauge invariance of $W_{\alpha}$ follows from the fact that $\bar{D}_{\dot{\alpha}} \Lambda=0$ by using the commutation relation $\left[\bar{D}^{2}, D_{\alpha}\right]=4 i\left(\sigma^{m} \bar{D}\right)_{\alpha} \partial_{m}$.

Returning to the case of non-linearly realized $\mathcal{N}=2$ supersymmetry, we note that the superfield $\Phi$ appearing in (4.19) and defined in 4.15) is not an $\mathcal{N}=1$ chiral superfield in the sense that $\bar{D}_{\dot{\alpha}} \Phi \neq 0$. However, one can show [9] that $\overline{\mathcal{D}}_{\dot{\alpha}} \Phi=0$ if $\bar{D}_{\dot{\alpha}} \tilde{\Phi}=0$. Thus, the condition on the gauge parameter $\Lambda$ has to be generalized to $\overline{\mathcal{D}}_{\dot{\alpha}} \Lambda=0$. This guarantees that the $\mathcal{N}=2$ chirality of $\Phi, \overline{\mathcal{D}}_{\dot{\alpha}} \Phi=0$, is preserved under gauge transformations.

An explicit computation of the commutation relation $\left[\overline{\mathcal{D}}^{2}, \mathcal{D}_{\alpha}\right]$ using the formulae given in the appendix yields

$$
\begin{aligned}
{\left[\overline{\mathcal{D}}^{2}, \mathcal{D}_{\alpha}\right]=} & 4 i D_{m}\left(\sigma^{m} \overline{\mathcal{D}}\right)_{\alpha}-4 i \kappa^{2} \mathcal{D}_{\alpha}\left(\Lambda_{g} \sigma^{m}\right)_{\dot{\gamma}} \overline{\mathcal{D}}_{\dot{\beta}} \bar{\Lambda}_{g}^{\dot{\gamma}} D_{m} \overline{\mathcal{D}}^{\dot{\beta}} \\
& -2 i \kappa^{2} \mathcal{D}_{\alpha}\left(\Lambda_{g} \sigma^{m}\right)_{\dot{\gamma}} \overline{\mathcal{D}}^{2} \bar{\Lambda}_{g}^{\dot{\gamma}} D_{m}+\mathcal{O}\left(\kappa^{4}\right)
\end{aligned}
$$

This implies

$$
\overline{\mathcal{D}}^{2} \mathcal{D}_{\alpha}\left(\Lambda-\Lambda^{\dagger}\right)=\left[\overline{\mathcal{D}}^{2}, \mathcal{D}_{\alpha}\right] \Lambda=-2 i \kappa^{2} \mathcal{D}_{\alpha}\left(\Lambda_{g} \sigma^{m}\right)_{\dot{\gamma}} \overline{\mathcal{D}}^{2} \bar{\Lambda}_{g}^{\dot{\gamma}} D_{m} \Lambda+\mathcal{O}\left(\kappa^{4}\right)
$$

As a consequence, $\mathcal{W}_{\alpha}^{0}$ as defined in 4.21 is not gauge invariant. But

$$
\mathcal{W}_{\alpha}^{\prime}=-\frac{1}{4}\left(\overline{\mathcal{D}}^{2} \mathcal{D}_{\alpha}-\kappa^{2} \mathcal{D}_{\alpha} \Lambda_{g}^{\beta} \overline{\mathcal{D}}^{2} \bar{\Lambda}_{g}^{\dot{\gamma}} \overline{\mathcal{D}}_{\dot{\gamma}} \mathcal{D}_{\beta}+\mathcal{O}\left(\kappa^{4}\right)\right) V
$$

is gauge invariant and still transforms according to the standard non-linear realization (4.16) up to order $\kappa^{2}$. Finally,

$$
\mathcal{W}_{\alpha}(x, \theta, \bar{\theta})=\mathcal{W}_{\alpha}^{\prime}\left(x^{m}-i \kappa^{2} \Lambda_{g} \sigma^{m} \bar{\Lambda}_{g}, \theta, \bar{\theta}\right)
$$

has the desired chiral transformation law (4.13).

Note that it is not possible to couple charged matter to the goldstino gauge multiplet. The term

$$
\mathcal{L}_{\text {gauge }}=\int d^{2} \theta d^{2} \bar{\theta} \hat{E} \Phi^{\dagger} e^{V_{g}} \Phi
$$

is not invariant, because of the shift in $V_{g}$. Here, $V_{g}$ is the vector superfield containing $\lambda_{g}$, i.e., $W_{g \alpha}=-\frac{1}{4} \bar{D}^{2} D_{\alpha} V_{g}$. From the transformation of $W_{g \alpha}$, eq. (4.4), we find

$$
\delta_{\eta}^{\star} V_{g}=\frac{2}{\kappa}\left(\bar{\theta} \bar{\theta}-\kappa^{2} \bar{X}\right) \theta \eta+\frac{2}{\kappa}\left(\theta \theta-\kappa^{2} X\right) \bar{\theta} \bar{\eta}
$$

Therefore, $\delta_{\eta}^{\star} \mathcal{L}_{\text {gauge }}=\left.\frac{2}{\kappa}(\eta D+\bar{\eta} \bar{D})\left(\hat{E} \Phi^{\dagger} e^{V_{g}} \Phi\right)\right|_{\theta=0}$.

This is not a problem for D-brane models because there is no matter that is charged under the goldstino $U(1)$ in such models. To see this, consider a stack of $N$ D-branes. The gauge symmetry of the theory on world-volume of the D-branes is of the form $\prod_{i} U\left(n_{i}\right)$, 
with $\sum_{i} n_{i}=N$. All matter fields are in bifundamental representations, $\left(n_{i}, \overline{n_{j}}\right)$, for some $i, j$ (this is the adjoint representation if $i=j$ ). The goldstino $U(1)$ is diagonally embedded in the D-brane gauge group, i.e., $U(1)_{\text {goldstino }}=\sum_{i} U_{i}(1)$, where $U_{i}(1)$ is the trace part of $U\left(n_{i}\right)$. As a consequence, all matter fields are neutral under $U(1)_{\text {goldstino. This general }}$ argument applies to D-branes in the bulk, which break $\mathcal{N}=8$ supersymmetry to $\mathcal{N}=4$, and to D-branes at an orbifold fixed point breaking either $\mathcal{N}=4$ supersymmetry to $\mathcal{N}=2$ or $\mathcal{N}=2$ supersymmetry to $\mathcal{N}=1$. In the case of extended supersymmetry on the Dbranes, there is more than one goldstino. The world-volume theory of a stack of $N$ Dbranes in the bulk, for example, is an $N=4$ super-Yang-Mills theory with gauge group

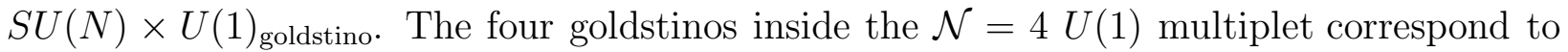
the four broken supersymmetries. The argument does not apply to D-branes at orientifold singularities. But supersymmetry breaking in orientifolds is not a partial breaking since bulk and (parallel) branes have the same amount of supersymmetry in such models.

For the same reason, it is not possible to couple a chiral superfield to the goldstino gauge kinetic terms. The Lagrangian

$$
\mathcal{L}=\int d^{2} \theta E_{L} \tilde{\Phi} \tilde{\Lambda}_{g} \tilde{\Lambda}_{g}+\int d^{2} \bar{\theta} E_{R} \tilde{\Phi}^{\dagger} \overline{\tilde{\Lambda}}_{g} \overline{\tilde{\Lambda}}_{g}
$$

transforms as $\delta_{\eta}^{\star} \mathcal{L}=\frac{1}{\kappa} \int d^{2} \theta \tilde{\Phi} W_{g} \eta+\frac{1}{\kappa} \int d^{2} \bar{\theta} \tilde{\Phi}^{\dagger} \bar{W}_{g} \bar{\eta}$, which is not a total derivative. This is puzzling because in string theory the gauge coupling constant is related to the expectation value of the dilaton and the coupling of the dilaton superfield to the brane gauge fields should be of the form (4.28).

\section{Coupling $\mathcal{N}=2$ multiplets to $\mathcal{N}=1$ matter}

\subsection{General formalism}

We now want to include fields on which $\mathcal{N}=2$ supersymmetry is realized linearly and determine their couplings to the $\mathcal{N}=1$ superfields that have no $\mathcal{N}=2$ partners. The method will be a straightforward generalization of the one discussed in section 3 . We work in the extended superspace spanned by the coordinates $(x, \theta, \bar{\theta}, \tilde{\theta}, \overline{\tilde{\theta}})$. Let $\mathcal{F}$ be an $\mathcal{N}=2$ superfield $^{3}$ which has the $\mathcal{N}=1$ superfield $F$ as its lowest component in the $\tilde{\theta}$ expansion, i.e.,

$$
\mathcal{F}=e^{\tilde{\theta} S+\overline{\tilde{\theta}} \bar{S}} F
$$

where $S, \bar{S}$ are the generators of the second supersymmetry and the action of $S, \bar{S}$ on $F$ is defined by $(\eta S+\bar{\eta} \bar{S}) F \equiv \delta_{\eta}^{\star} F$.

The effect of a supersymmetry transformation acting on $\mathcal{F}$ is a shift in extended superspace. For a transformation of the second supersymmetry, one has

$$
\mathcal{F}^{\prime}(x, \theta, \bar{\theta}, \tilde{\theta}, \overline{\tilde{\theta}})=e^{\eta S+\bar{\eta} \bar{S}} \mathcal{F}(x, \theta, \bar{\theta}, \tilde{\theta}, \overline{\tilde{\theta}})
$$

\footnotetext{
${ }^{3}$ We restrict ourselves to realizations of the $\mathcal{N}=2$ supersymmetry algebra without central charge. That is, we assume $\left\{Q_{\alpha}, S_{\beta}\right\}=0$.
} 


$$
\begin{aligned}
& =\mathcal{F}\left(x^{m}-i\left(\eta \sigma^{m} \overline{\tilde{\theta}}-\tilde{\theta} \sigma^{m} \bar{\eta}\right), \theta, \bar{\theta}, \tilde{\theta}+\eta, \overline{\tilde{\theta}}+\bar{\eta}\right) \\
& =\mathcal{F}\left(x^{\prime}, \theta^{\prime}, \bar{\theta}^{\prime}, \tilde{\theta}^{\prime}, \overline{\tilde{\theta}^{\prime}}\right)
\end{aligned}
$$

The goldstino superfield can be viewed as a hypersurface in extended superspace defined by

$$
\tilde{\theta}=-\kappa \Lambda_{g}(x, \theta, \bar{\theta})
$$

The requirement that this hypersurface be invariant under transformations of the second supersymmetry,

$$
\tilde{\theta}^{\prime}(x, \theta, \bar{\theta})=\tilde{\theta}\left(x^{\prime}, \theta^{\prime}, \bar{\theta}^{\prime}\right) \quad \Longrightarrow \quad-\kappa \Lambda_{g}^{\prime}(x, \theta, \bar{\theta})+\eta=-\kappa \Lambda_{g}\left(x^{\prime}, \theta^{\prime}, \bar{\theta}^{\prime}\right),
$$

implies (for infinitesimal $\eta$ )

$$
\Lambda_{g}^{\prime}(x, \theta, \bar{\theta})=\Lambda_{g}(x, \theta, \bar{\theta})+\frac{1}{\kappa} \eta+i \kappa\left(\eta \sigma^{m} \bar{\Lambda}_{g}(x, \theta, \bar{\theta})-\Lambda_{g}(x, \theta, \bar{\theta}) \sigma^{m} \bar{\eta}\right) \partial_{m} \Lambda_{g}(x, \theta, \bar{\theta}),
$$

which coincides with the transformation law (4.1).

Now, consider the $\mathcal{N}=2$ superfield $\mathcal{F}$ restricted to the hypersurface (5.3),

$$
\hat{F}(x) \equiv \mathcal{F}\left(x, \theta, \bar{\theta},-\kappa \Lambda_{g}(x, \theta, \bar{\theta}),-\kappa \bar{\Lambda}_{g}(x, \theta, \bar{\theta})\right)
$$

Under an infinitesimal supersymmetry transformation it varies as

$$
\begin{aligned}
\delta_{\eta}^{\star} \hat{F}(x, \theta, \bar{\theta}) & =i \kappa\left(\eta \sigma^{m} \bar{\Lambda}_{g}(x, \theta, \bar{\theta})-\Lambda_{g}(x, \theta, \bar{\theta}) \sigma^{m} \bar{\eta}\right) \partial_{m} \hat{F}(x, \theta, \bar{\theta}) \\
& =-\kappa V_{\eta}^{m} \partial_{m} \hat{F}(x, \theta, \bar{\theta})
\end{aligned}
$$

The composite superfield $\hat{F}$ reduces to $F$ in the limit $\kappa \rightarrow 0$ and transforms according to the standard non-linear realization (4.16).

It is now clear how to couple the $\mathcal{N}=1$ superfield $F$ that is the lowest component of an $\mathcal{N}=2$ superfield $\mathcal{F}$ to $\mathcal{N}=1$ superfields $\Phi_{i}$ that have no $\mathcal{N}=2$ partners in such a way that all interactions are invariant under the full $\mathcal{N}=2$ supersymmetry. If we know how $F$ couples to the $\Phi_{i}$ in the limit $\kappa \rightarrow 0$, then the $\mathcal{N}=2$ completion of these couplings is obtained by replacing $F$ by $\hat{F}$.

Let us now consider the chiral version of non-linear supersymmetry. A chiral $\mathcal{N}=2$ superfield $\boldsymbol{\Phi}$ is a function of $x_{L}, \theta$ and $\tilde{\theta}$ only, where

$$
x_{L}^{m}=x^{m}+i \theta \sigma^{m} \bar{\theta}+i \tilde{\theta} \sigma^{m} \overline{\tilde{\theta}} .
$$

In the $\left(x_{L}, \theta, \tilde{\theta}\right)$ basis, the $\tilde{\theta}$ expansion of $\boldsymbol{\Phi}$ is simply given by

$$
\Phi=e^{\tilde{\theta} S} \Phi=\Phi+\sqrt{2} \tilde{\theta} \Psi+\tilde{\theta} \tilde{\theta} \boldsymbol{F}
$$

where $\Phi, \Psi_{\alpha}, \boldsymbol{F}$ are chiral $\mathcal{N}=1$ superfields. 
A supersymmetry transformation acting on $\boldsymbol{\Phi}$ yields

$$
\begin{aligned}
\boldsymbol{\Phi}^{\prime}\left(x_{L}, \theta, \tilde{\theta}\right) & =e^{\eta S+\bar{\eta} \bar{S}} \boldsymbol{\Phi}\left(x_{L}, \theta, \tilde{\theta}\right) \\
& =e^{(\tilde{\theta}+\eta) S-\left(2 \tilde{\theta} \sigma^{m} \bar{\eta}+\eta \sigma^{m} \bar{\eta}\right) P_{m}} e^{\bar{\eta} \bar{S}} \Phi\left(x_{L}, \theta\right) \\
& =\boldsymbol{\Phi}\left(x_{L}^{m}+2 i \tilde{\theta} \sigma^{m} \bar{\eta}+i \eta \sigma^{m} \bar{\eta}, \theta, \tilde{\theta}+\eta\right)=\boldsymbol{\Phi}\left(x_{L}^{\prime}, \theta^{\prime}, \tilde{\theta}^{\prime}\right)
\end{aligned}
$$

where we used that $\bar{S}_{\dot{\alpha}} \Phi=0$.

The chiral goldstino superfield $\tilde{\Lambda}_{g}$ can be viewed as a hypersurface in chiral extended superspace defined by

$$
\tilde{\theta}=-\kappa \tilde{\Lambda}_{g}\left(x_{L}, \theta\right) .
$$

The requirement that this hypersurface be invariant under supersymmetry transformations,

$$
\tilde{\theta}^{\prime}\left(x_{L}, \theta\right)=\tilde{\theta}\left(x_{L}^{\prime}, \theta^{\prime}\right) \quad \Longrightarrow \quad-\kappa \tilde{\Lambda}_{g}^{\prime}\left(x_{L}, \theta\right)+\eta=-\kappa \Lambda_{g}\left(x_{L}^{\prime}, \theta^{\prime}\right),
$$

implies (for infinitesimal $\eta$ )

$$
\tilde{\Lambda}_{g}^{\prime}\left(x_{L}, \theta\right)=\tilde{\Lambda}_{g}\left(x_{L}, \theta\right)+\frac{1}{\kappa} \eta-2 i \kappa \tilde{\Lambda}_{g}\left(x_{L}, \theta\right) \sigma^{m} \bar{\eta} \partial_{m} \tilde{\Lambda}_{g}\left(x_{L}, \theta\right),
$$

which coincides with the transformation law (4.2). As a byproduct, we find the relation $\tilde{\Lambda}_{g}\left(x_{L}, \theta\right)=\Lambda_{g}(x, \theta, \bar{\theta})$, where $x_{L}^{m}=x^{m}+i \theta \sigma^{m} \bar{\theta}+i \kappa^{2} \tilde{\Lambda}_{g}\left(x_{L}, \theta\right) \sigma^{m} \tilde{\tilde{\Lambda}}_{g}\left(x_{L}, \theta\right)$. The transformation of an $\mathcal{N}=1$ chiral superfield $\tilde{\Phi}\left(x_{L}, \theta\right)$ in the chiral goldstino background is determined by the condition that $\tilde{\Phi}\left(x_{L}, \theta\right)$ be well-defined on the hypersurface (5.11), i.e., $\tilde{\Phi}^{\prime}\left(x_{L}, \theta\right)=\tilde{\Phi}\left(x_{L}^{\prime}, \theta\right)$. This implies the transformation law (4.13).

Now, consider the $\mathcal{N}=2$ superfield $\boldsymbol{\Phi}$ restricted to the hypersurface (5.11),

$$
\hat{\Phi}\left(x_{L}, \theta\right) \equiv \boldsymbol{\Phi}\left(x_{L}, \theta,-\kappa \tilde{\Lambda}_{g}\left(x_{L}, \theta\right)\right)
$$

Under an infinitesimal supersymmetry transformation it varies as

$$
\delta_{\eta}^{\star} \hat{\Phi}\left(x_{L}, \theta\right)=-2 i \kappa \tilde{\Lambda}_{g}\left(x_{L}, \theta\right) \sigma^{m} \bar{\eta} \partial_{m} \hat{\Phi}\left(x_{L}, \theta\right)
$$

which is the chiral standard non-linear transformation law (4.13).

\subsection{Coupling an $\mathcal{N}=2$ vector to $\mathcal{N}=1$ multiplets}

Let us work out explicitly the couplings of an Abelian $\mathcal{N}=2$ vector multiplet to $\mathcal{N}=1$ matter. An $\mathcal{N}=2$ vector can be decomposed in an $\mathcal{N}=1$ vector $V$ and an $\mathcal{N}=1$ chiral multiplet $\Phi$.

$$
\begin{aligned}
V & =-\theta \sigma^{m} \bar{\theta} A_{m}+i \theta \theta \bar{\theta} \bar{\lambda}^{(1)}-i \bar{\theta} \bar{\theta} \theta \lambda^{(1)}+\frac{1}{2} \theta \theta \bar{\theta} \bar{\theta} D \\
\Phi & =\phi+i \theta \sigma^{m} \bar{\theta} \partial_{m} \phi+\frac{1}{4} \theta \theta \bar{\theta} \bar{\theta} \square \phi+\sqrt{2} \theta \lambda^{(2)}-\frac{i}{\sqrt{2}} \theta \theta \partial_{m} \lambda^{(2)} \sigma^{m} \bar{\theta}+\theta \theta F
\end{aligned}
$$


The field strength superfield $W_{\alpha}=-\frac{1}{4} \bar{D}^{2} D_{\alpha} V$ has the component expansion

$$
\begin{aligned}
W_{\alpha}= & -i \lambda_{\alpha}^{(1)}+\theta_{\alpha} D-i\left(\sigma^{m n} \theta\right)_{\alpha} F_{m n}+\theta \sigma^{m} \bar{\theta} \partial_{m} \lambda_{\alpha}^{(1)}+\theta \theta\left(\sigma^{m} \partial_{m} \bar{\lambda}^{(1)}\right)_{\alpha} \\
& -\frac{1}{2} \theta \theta\left(\sigma^{m} \bar{\theta}\right)_{\alpha}\left(i \partial_{m} D-\partial^{n} F_{m n}\right)-\frac{i}{4} \theta \theta \bar{\theta} \bar{\theta} \square \lambda_{\alpha}^{(1)} .
\end{aligned}
$$

The supersymmetry transformations are (see, e.g., [30])

$$
\begin{aligned}
\delta_{\xi} \phi & =\sqrt{2}\left(\xi^{(1)} \lambda^{(2)}-\xi^{(2)} \lambda^{(1)}\right) \\
\delta_{\xi} \lambda^{(1)} & =\sigma^{m n} \xi^{(1)} F_{m n}+i \xi^{(1)} D-i \sqrt{2} \sigma^{m} \bar{\xi}^{(2)} \partial_{m} \phi-\sqrt{2} \xi^{(2)} F^{\dagger}, \\
\delta_{\xi} \lambda^{(2)} & =\sigma^{m n} \xi^{(2)} F_{m n}-i \xi^{(2)} D+i \sqrt{2} \sigma^{m} \bar{\xi}^{(1)} \partial_{m} \phi+\sqrt{2} \xi^{(1)} F \\
\delta_{\xi} A_{m} & =i \xi^{(1)} \sigma_{m} \bar{\lambda}^{(1)}-i \lambda^{(1)} \sigma_{m} \bar{\xi}^{(1)}+i \xi^{(2)} \sigma_{m} \bar{\lambda}^{(2)}-i \lambda^{(2)} \sigma_{m} \bar{\xi}^{(2)}, \\
\delta_{\xi} D & =-\xi^{(1)} \sigma^{m} \partial_{m} \bar{\lambda}^{(1)}-\partial_{m} \lambda^{(1)} \sigma^{m} \bar{\xi}^{(1)}+\xi^{(2)} \sigma^{m} \partial_{m} \bar{\lambda}^{(2)}+\partial_{m} \lambda^{(2)} \sigma^{m} \bar{\xi}^{(2)}, \\
\delta_{\xi} F & =i \sqrt{2}\left(\bar{\xi}^{(1)} \bar{\sigma}^{m} \partial_{m} \lambda^{(2)}+\partial_{m} \bar{\lambda}^{(1)} \bar{\sigma}^{m} \xi^{(2)}\right) .
\end{aligned}
$$

One finds that the supersymmetry algebra closes on $\Phi$, but on $V$ it closes only up to a gauge transformation,

$$
\begin{aligned}
{\left[\delta_{\eta}^{\star}, \delta_{\xi}^{\star}\right] \Phi } & =-2 i\left(\eta \sigma^{m} \bar{\xi}-\xi \sigma^{m} \bar{\eta}\right) \partial_{m} \Phi \\
{\left[\delta_{\eta}^{\star}, \delta_{\xi}^{\star}\right] V } & =-2 i\left(\eta \sigma^{m} \bar{\xi}-\xi \sigma^{m} \bar{\eta}\right) \partial_{m} V+\theta \sigma^{n} \bar{\theta}\left(-2 i\left(\eta \sigma^{m} \bar{\xi}-\xi \sigma^{m} \bar{\eta}\right) \partial_{n} A_{m}\right)
\end{aligned}
$$

We are interested in two different kinds of couplings. Firstly, the vector superfield $V$ inside the $\mathcal{N}=2$ vector can couple to $\mathcal{N}=1$ chiral superfields $\tilde{\Phi}_{i}$. Secondly, the chiral superfield $\Phi$ inside the $\mathcal{N}=2$ vector can couple to $\mathcal{N}=1$ chiral superfields $\tilde{\Phi}_{i}$ and to $\mathcal{N}=1$ field strength superfields $\tilde{W}_{\alpha}^{a}$.

Let us start by constructing the $\mathcal{N}=2$ completion of the $\mathcal{N}=1$ supersymmetric gauge coupling term $\int d^{2} \theta d^{2} \bar{\theta} \tilde{\Phi}^{\dagger} e^{V} \tilde{\Phi}$. According to the general formalism, developed above, we need to compute the $\tilde{\theta}$ expansion of an $\mathcal{N}=2$ superfield $\mathcal{V}$ that has the $\mathcal{N}=1$ vector $V$ as its lowest component. Using

$$
\begin{aligned}
\delta_{\eta}^{\star} V & =-i \theta \sigma^{m} \bar{\theta}\left(\eta \sigma_{m} \bar{D} \Phi^{\dagger}+\bar{\eta} \bar{\sigma}_{m} D \Phi\right), \\
\delta_{\eta}^{\star} \Phi & =-i \sqrt{2} W \eta, \\
\delta_{\eta}^{\star} W_{\alpha} & =\frac{i}{\sqrt{2}} \bar{\eta} \bar{D} D_{\alpha} \Phi-\frac{i}{2 \sqrt{2}} \eta_{\alpha} \bar{D}^{2} \Phi^{\dagger},
\end{aligned}
$$

we find,

$$
\begin{aligned}
\mathcal{V} & =e^{\tilde{\theta} S+\overline{\tilde{\theta}} \bar{S}} V \\
& =V-\theta \sigma^{m} \bar{\theta}\left[i\left(\overline{\tilde{\theta}} \bar{\sigma}_{m} D \Phi+\tilde{\theta} \sigma_{m} \bar{D} \Phi^{\dagger}\right)-\frac{1}{2 \sqrt{2}} \tilde{\theta} \sigma^{n} \overline{\tilde{\theta}}\left(D \sigma_{m} \bar{\sigma}_{n} W+\bar{D} \bar{\sigma}_{m} \sigma_{n} \bar{W}\right)\right.
\end{aligned}
$$




$$
\begin{aligned}
& -\tilde{\theta} \tilde{\theta} \overline{\tilde{\theta}}\left(\bar{\sigma}_{m n} \bar{D} \partial^{n} \Phi^{\dagger}-\frac{1}{6} \bar{D} \partial_{m} \Phi^{\dagger}\right)-\overline{\tilde{\theta}} \overline{\tilde{\theta}} \tilde{\theta}\left(\sigma_{m n} D \partial^{n} \Phi-\frac{1}{6} D \partial_{m} \Phi\right) \\
& \left.+\frac{i}{4 \sqrt{2}} \tilde{\theta} \tilde{\theta} \overline{\tilde{\theta}} \overline{\tilde{\theta}}\left(D \sigma_{m n} \partial^{n} W-\bar{D} \bar{\sigma}_{m n} \partial^{n} \bar{W}\right)\right] .
\end{aligned}
$$

Then, we define

$$
\left.\hat{V} \equiv \mathcal{V}\right|_{\tilde{\theta}=-\kappa \Lambda_{g}}
$$

In the non-supersymmetric case, section 3.3, we saw that the commutation relation $\left[\delta_{\eta}, \delta_{\xi}\right]=-2 i\left(\eta \sigma^{n} \bar{\xi}-\xi \sigma^{n} \bar{\eta}\right) F_{n m}$ implied the transformation law $\delta_{\xi} \hat{A}_{m}=-\kappa v_{\xi}^{n} \hat{F}_{n m}$. It is easy to generalize this to the present case. Taking into account the extra term in the commutator $\left[\delta_{\eta}^{\star}, \delta_{\xi}^{\star}\right] V$, eq. (5.19), one finds that eq. (5.7) is modified to

$$
\delta_{\eta}^{\star} \hat{V}=-\kappa V_{\eta}^{m} \partial_{m} \hat{V}-\kappa V_{\eta}^{m} \theta \sigma^{n} \bar{\theta} \partial_{n} \hat{A}_{m}
$$

where $\hat{A}_{m}$ is the coefficient of $-\theta \sigma^{m} \bar{\theta}$ in the superspace expansion of $\hat{V}$. This coincides with the expression for $\hat{A}_{m}$ given in eq. (3.27).

We want to couple $\hat{V}$ to $\mathcal{N}=1$ chiral multiplets $\tilde{\Phi}_{i}$ that carry charges $q_{i}$ under the $U(1)$ gauge symmetry of the vector $V$. To achieve this, we define

$$
\Phi_{i}(x, \theta, \bar{\theta})=\tilde{\Phi}_{i}\left(x_{L}, \theta\right), \quad \text { where } x_{L}^{m}=x^{m}+i \theta \sigma^{m} \bar{\theta}+i \kappa^{2} \Lambda_{g} \sigma^{m} \bar{\Lambda}_{g},
$$

and modify the $\Phi_{i}$ transformation law (4.16) to

$$
\delta_{\eta}^{\star} \Phi_{i}=-\kappa V_{\eta}^{m}\left(\partial_{m} \Phi_{i}-\frac{1}{2} q_{i} \theta \sigma^{n} \bar{\theta} \partial_{n} \hat{A}_{m} \Phi_{i}\right),
$$

Knowing how $\hat{V}$ and $\Phi_{i}$ transform under the second supersymmetry, we conclude that the Lagrangian

$$
\begin{aligned}
\mathcal{L} & =\int d^{2} \theta d^{2} \bar{\theta} \hat{E} \Phi_{i}^{\dagger} e^{q_{i} \hat{V}} \Phi_{i} \\
& =\int d^{2} \theta d^{2} \bar{\theta}\left(1+i \kappa q_{i} \theta \sigma^{m} \bar{\theta}\left(\bar{\Lambda}_{g} \bar{\sigma}_{m} D \Phi+\Lambda_{g} \sigma_{m} \bar{D} \Phi^{\dagger}\right)\right) \tilde{\Phi}_{i}^{\dagger} e^{q_{i} V} \tilde{\Phi}_{i}+\mathcal{O}\left(\kappa^{2}\right)
\end{aligned}
$$

is invariant up to a total derivative.

Next, let us construct the $\mathcal{N}=2$ completion of the $\mathcal{N}=1$ supersymmetric couplings $\int d^{2} \theta\left(\Phi \tilde{W}^{a} \tilde{W}^{a}+\mathcal{P}\left(\Phi, \tilde{\Phi}_{i}\right)\right)$, where $\Phi$ is the $\mathcal{N}=1$ chiral superfield inside the $\mathcal{N}=2$ vector and $\mathcal{P}$ is an arbitrary analytic function. The $\tilde{\theta}$ expansion of the $\mathcal{N}=2$ superfield $\boldsymbol{\Phi}$ that has $\Phi$ as its lowest component is much simpler than the $\tilde{\theta}$ expansion of $\mathcal{V}$. Since $\bar{S}_{\dot{\alpha}} \Phi=0$, $\boldsymbol{\Phi}$ is an $\mathcal{N}=2$ chiral superfield. One finds

$$
\Phi=\Phi-i \sqrt{2} \tilde{\theta} W-\frac{1}{4} \tilde{\theta} \tilde{\theta} \bar{D}^{2} \Phi^{\dagger} .
$$

This implies that

$$
\hat{\Phi} \equiv \Phi+i \sqrt{2} \kappa \tilde{\Lambda}_{g} W-\frac{1}{4} \kappa^{2} \tilde{\Lambda}_{g} \tilde{\Lambda}_{g} \bar{D}^{2} \Phi^{\dagger}
$$

transforms according to the chiral standard non-linear realization (4.13) and

$$
\mathcal{L}=\int d^{2} \theta E_{L}\left(\hat{\Phi} \tilde{\mathcal{W}}^{a} \tilde{\mathcal{W}}^{a}+\mathcal{P}\left(\hat{\Phi}, \tilde{\Phi}_{i}\right)\right)+\text { h.c. }
$$

is invariant up to a total derivative. 


\section{Pseudo-Supersymmetry}

The formalism explained in the previous sections is well-suited to analyze a class of very interesting supersymmetry breaking scenarios that arise naturally in string theory. In models containing D-branes and anti-D-branes (e.g., [31]) or intersecting D-branes (e.g., [7), different sectors of the theory break different halves of supersymmetry, thus breaking supersymmetry completely but in a non-local way. All broken supersymmetries are non-linearly realized. Such a scenario is called pseudo-supersymmetry.

Let us consider a toy model containing three sectors, a bulk sector with $\mathcal{N}=2$ supersymmetry and two boundary sectors preserving the first and second of the two bulk supersymmetries, respectively. We concentrate on cases where there is only an $\mathcal{N}=2$ vector $\left(A_{m}, \lambda^{(1)}, \lambda^{(2)}, \phi\right)$ in the bulk that couples to chiral multiplets $\tilde{\Phi}^{(1)}, \tilde{\Phi}^{(2)}$ and gauge field strengths $\tilde{W}^{(1)}, \tilde{W}^{(2)}$ on the boundaries. Here $\tilde{\Phi}^{(1)}, \tilde{W}^{(1)}$ are chiral superfields with respect to the first supersymmetry and $\tilde{\Phi}^{(2)}, \tilde{W}^{(2)}$ are chiral superfields with respect to the second supersymmetry.

$$
\begin{aligned}
\tilde{\Phi}^{(1)} & =\phi^{(1)}+\sqrt{2} \theta \psi^{(1)}+\theta \theta F^{(1)} \\
\tilde{W}_{\alpha}^{(1)} & =-i \tilde{\lambda}_{\alpha}^{(1)}+\theta_{\alpha} D^{(1)}-i\left(\sigma^{m n} \theta\right)_{\alpha} F_{m n}^{(1)}+\theta \theta\left(\sigma^{m} \partial_{m} \overline{\tilde{\lambda}}^{(1)}\right)_{\alpha} \\
\tilde{\Phi}^{(2)} & =\phi^{(2)}+\sqrt{2} \tilde{\theta} \psi^{(2)}+\tilde{\theta} \tilde{\theta} F^{(2)} \\
\tilde{W}_{\alpha}^{(2)} & =-i \tilde{\lambda}_{\alpha}^{(2)}+\tilde{\theta}_{\alpha} D^{(2)}-i\left(\sigma^{m n} \tilde{\theta}\right)_{\alpha} F_{m n}^{(2)}+\tilde{\theta} \tilde{\theta}\left(\sigma^{m} \partial_{m} \overline{\tilde{\lambda}}^{(2)}\right)_{\alpha}
\end{aligned}
$$

The $\mathcal{N}=2$ vector $\left(A_{m}, \lambda^{(1)}, \lambda^{(2)}, \phi\right)$ can be either split into two $\mathcal{N}=1$ superfields with respect to the first supersymmetry. These are the vector $V$ and the chiral multiplet $\Phi$ defined in (5.16).

$$
\begin{aligned}
& V=-\theta \sigma^{m} \bar{\theta} A_{m}+i \theta \theta \bar{\theta} \bar{\lambda}^{(1)}-i \bar{\theta} \bar{\theta} \theta \lambda^{(1)}+\frac{1}{2} \theta \theta \bar{\theta} \bar{\theta} D \\
& \Phi=\phi+i \theta \sigma^{m} \bar{\theta} \partial_{m} \phi+\frac{1}{4} \theta \theta \bar{\theta} \bar{\theta} \square \phi+\sqrt{2} \theta \lambda^{(2)}-\frac{i}{\sqrt{2}} \theta \theta \partial_{m} \lambda^{(2)} \sigma^{m} \bar{\theta}+\theta \theta F
\end{aligned}
$$

Or we can split the $\mathcal{N}=2$ vector into two $\mathcal{N}=1$ superfields with respect to the second supersymmetry.

$$
\begin{aligned}
& V^{\prime}=-\tilde{\theta} \sigma^{m} \overline{\tilde{\theta}} A_{m}+i \tilde{\theta} \tilde{\theta} \overline{\tilde{\theta}} \bar{\lambda}^{(2)}-i \overline{\tilde{\theta}} \tilde{\tilde{\theta}} \tilde{\lambda}{ }^{(2)}-\frac{1}{2} \tilde{\theta} \tilde{\theta} \tilde{\tilde{\theta}} \overline{\tilde{\theta}} D \\
& \Phi^{\prime}=\phi+i \tilde{\theta} \sigma^{m} \overline{\tilde{\theta}} \partial_{m} \phi+\frac{1}{4} \tilde{\theta} \tilde{\theta} \tilde{\tilde{\theta}} \overline{\tilde{\theta}} \square \phi-\sqrt{2} \tilde{\theta} \lambda^{(1)}+\frac{i}{\sqrt{2}} \tilde{\theta} \tilde{\theta} \partial_{m} \lambda^{(1)} \sigma^{m} \overline{\tilde{\theta}}-\tilde{\theta} \tilde{\theta} F^{\dagger}
\end{aligned}
$$

There are two goldstino superfields $\Lambda_{g}$ and $\Lambda_{g}^{\prime}$ in this class of models. $\Lambda_{g}(x, \theta, \bar{\theta})$ is associated with the breaking of the second supersymmetry and $\Lambda_{g}^{\prime}(x, \tilde{\theta}, \overline{\tilde{\theta}})$ is associated with the breaking of the first supersymmetry. In complete analogy to the partially broken supersymmetry discussed above, $\Lambda_{g}^{\prime}$ is related to the chiral superfield $\tilde{\Lambda}_{g}^{\prime}$ and the field strength

\footnotetext{
${ }^{4} \mathrm{~A}$ related situation with non-linearly realized $\mathcal{N}=2$ supergravity is analyzed in 10 .
} 
superfield $W_{g}^{\prime}$ through eqs. (4.15), (4.10), (4.6) after putting primes on all fields and replacing $\theta$ by $\tilde{\theta}$.

To build an $\mathcal{N}=2$ invariant action, we construct chiral densities $E_{L}^{(1)}$ and $E_{L}^{(2)}$ from $W_{g}$ and $W_{g}^{\prime}$, respectively, as in (4.11), we form real densities $\hat{E}^{(1)}$ and $\hat{E}^{(2)}$ from $\Lambda_{g}$ and $\Lambda_{g}^{\prime}$, respectively, as in (4.17), and we define $\Phi^{(1)}, \Phi^{(2)}$ from $\tilde{\Phi}^{(1)}, \tilde{\Phi}^{(2)}$ as in (5.24). Furthermore, we define the field strength superfields $\tilde{\mathcal{W}}^{(1)}$ and $\tilde{\mathcal{W}}^{(2)}$ that transform covariantly under the second and first supersymmetry, respectively, as in (4.24), (4.25). Finally, we need to construct the composite superfields $\hat{V}, \hat{\Phi}$ as in (5.22), (5.21), (5.28), and similarly for $\hat{V}^{\prime}, \hat{\Phi}^{\prime}$. To obtain $\hat{V}^{\prime}, \hat{\Phi}^{\prime}$, one first computes the $\mathcal{N}=2$ superfields $\mathcal{V}^{\prime}, \Phi^{\prime}$ that have $V^{\prime}, \Phi^{\prime}$ as their lowest component in the $\theta$ expansion and then replaces $\theta \rightarrow-\kappa^{\prime} \Lambda_{g}^{\prime}$. Here, we assumed that the first supersymmetry is broken at a scale $\kappa^{\prime-1 / 2}$ and that $\Lambda_{g}^{\prime}$ transforms as in (4.1) but with $\kappa$ replaced by $\kappa^{\prime}$.

It is now straightforward to write down an $\mathcal{N}=2$ invariant Lagrangian for our toy model:

$$
\mathcal{L}=\mathcal{L}_{1}+\mathcal{L}_{2}+\mathcal{L}_{\text {bulk }}
$$

where

$$
\begin{aligned}
\mathcal{L}_{1}= & \int d^{2} \theta d^{2} \bar{\theta} \hat{E}^{(1)} \Phi^{(1) \dagger} e^{\hat{V}} \Phi^{(1)} \\
& +\int d^{2} \theta E_{L}^{(1)}\left((a+b \hat{\Phi}) \tilde{\mathcal{W}}^{(1)} \tilde{\mathcal{W}}^{(1)}+\mathcal{P}\left(\hat{\Phi}, \tilde{\Phi}^{(1)}\right)+\tilde{\Lambda}_{g} \tilde{\Lambda}_{g}\right)+\text { h.c. }, \\
\mathcal{L}_{2}= & \int d^{2} \tilde{\theta} d^{2} \overline{\tilde{\theta}} \hat{E}^{(2)} \Phi^{(2) \dagger} e^{\hat{V}^{\prime}} \Phi^{(2)} \\
& +\int d^{2} \tilde{\theta} E_{L}^{(2)}\left(\left(a^{\prime}+b^{\prime} \hat{\Phi}^{\prime}\right) \tilde{\mathcal{W}}^{(2)} \tilde{\mathcal{W}}^{(2)}+\mathcal{P}^{\prime}\left(\hat{\Phi}^{\prime}, \tilde{\Phi}^{(2)}\right)+\tilde{\Lambda}_{g}^{\prime} \tilde{\Lambda}_{g}^{\prime}\right)+\text { h.c. }, \\
\mathcal{L}_{\text {bulk }}= & \int d^{2} \theta d^{2} \bar{\theta} \Phi^{\dagger} e^{V} \Phi+\frac{1}{4} \int d^{2} \theta W W+\frac{1}{4} \int d^{2} \bar{\theta} \bar{W} \bar{W},
\end{aligned}
$$

and $a, b, a^{\prime}, b^{\prime}$ are undetermined coupling constants. A non-trivial superpotential $\mathcal{P}$ or $\mathcal{P}^{\prime}$ is possible only if either the boundary fields $\tilde{\Phi}^{(1)}, \tilde{\Phi}^{(2)}$ transform in real representations of the bulk gauge symmetry or if there are several oppositely charged fields on each boundary.

It is interesting to note that the interactions (6.5) preserve a $U(1)_{R}$ subgroup of the $S U(2)_{R}$ symmetry that is present in the $\mathcal{N}=2$ theory. Under this $U(1)_{R}$ symmetry the fields carry charges as shown in table 1. This implies that the superfields $V, \Phi, V^{\prime}, \Phi^{\prime}, \hat{V}$, $\hat{\Phi}, \hat{V}^{\prime}, \hat{\Phi}^{\prime}, E_{L}^{(i)}$ and $\hat{E}^{(i)}$ all have R-charge 0 , the superfields $\Lambda_{g}, \tilde{\mathcal{W}}^{(1)}, W$ have R-charge 1 and the superfields $\Lambda_{g}^{\prime}, \tilde{\mathcal{W}}^{(2)}, W^{\prime}$ have R-charge -1 .

This toy model is very similar to open string models of intersecting D-branes. Consider three stacks of D-branes at angles that intersect each other. Choose one of the stacks, say the third, and call it the bulk sector. Imagine that an orbifold compactification breaks supersymmetry down to $\mathcal{N}=2$ on each of the three stacks of D-branes, when considered separately. But only the first supersymmetry is preserved at the intersection of first with the third stack, and only the second supersymmetry is preserved at the intersection of the second with the third stack. These intersections are the boundary sectors of our toy model. 


\begin{tabular}{|c|c|c|c|c|c|c|c|c|c|c|c|c|}
\hline field & $\theta$ & $\tilde{\theta}$ & $d^{2} \theta$ & $d^{2} \tilde{\theta}$ & $\lambda_{g}$ & $\lambda_{g}^{\prime}$ & $\phi$ & $A_{m}$ & $\lambda^{(1)}$ & $\lambda^{(2)}$ & $\tilde{\lambda}^{(1)}$ & $\tilde{\lambda}^{(2)}$ \\
\hline R-charge & 1 & -1 & -2 & 2 & 1 & -1 & 0 & 0 & 1 & -1 & 1 & -1 \\
\hline
\end{tabular}

Table 1: R-charges of the Grassmann variables $\theta, \tilde{\theta}$, the goldstinos $\lambda_{g}, \lambda_{g}^{\prime}$, the components of the bulk vector multiplet $\left(\phi, A_{m}, \lambda^{(1)}, \lambda^{(2)}\right)$ and the boundary gauginos $\tilde{\lambda}^{(1)}, \tilde{\lambda}^{(2)}$.

In models of intersecting branes, all gauge fields live in the bulk and the chiral matter fields are confined to the intersections. Thus $a=b=a^{\prime}=b^{\prime}=0$ in such models.

Supersymmetry is completely broken in the toy model described by the above Lagrangian because $\mathcal{L}_{1}$ and $\mathcal{L}_{2}$ preserve different halves of the bulk $\mathcal{N}=2$ supersymmetry. What makes this supersymmetry breaking scenario very interesting is that any two of the three subsectors $\mathcal{L}_{1}, \mathcal{L}_{2}, \mathcal{L}_{\text {bulk }}$ are supersymmetric when considered separately from the third sector. That is obvious for $\mathcal{L}_{1}+\mathcal{L}_{\text {bulk }}$ and $\mathcal{L}_{2}+\mathcal{L}_{\text {bulk. }}$. For $\mathcal{L}_{1}+\mathcal{L}_{2}$, one has to set all bulk fields to zero and substitute $\tilde{\theta} \rightarrow \theta$ in $\mathcal{L}_{2}$. As a consequence, only interactions that involve fields from all three sectors can generate mass splittings for the components of the supersymmetric multiplets. Such interactions only arise at two loops [7]. This implies that the supersymmetric nonrenormalization theorems still apply at one loop. Thus there are no mass splittings and no quadratic divergences at one loop. The scalar masses squared arising at two loops are expected to be $\sim(g / 4 \pi)^{4} M^{2}$, where $g$ is the gauge coupling of the bulk vector and $M$ is the cut-off scale of the effective field theory. . A non-vanishing vacuum energy oly arises at three loops. This is similar to a two-site moose model studied in [33].

The fermion masses are protected to all orders in perturbation theory by the $U(1)_{R}$ symmetry mentioned above. Generically, this $U(1)_{R}$ is broken down to a discrete subgroup by a quantum anomaly, but this discrete subgroup suffices to forbid fermion mass terms (assuming they are not already present at tree-level). However, the $U(1)_{R}$ is broken explicitly, when the model is coupled to gravity. This is because the supercurrents corresponding to the two supersymmetries do not have well-defined R-charges in the goldstino background. The supercurrents are polynomials in the fields and their first derivatives. It turns out that different terms in these polynomials have different R-charges. To see this, first consider the sector $\mathcal{L}_{1}+\mathcal{L}_{\text {bulk. }}$. Note that the goldstino transformation law (4.1) is only compatible with the charge assignments of table 1 if we assign R-charge 1 to the supersymmetry variation parameter $\eta$ whereas the vector transformation law (5.18) is only compatible with the charge assignments of table 1 if we assign R-charge -1 to the supersymmetry variation parameter $\eta=\xi^{(2)}$. This apparent mismatch is not a problem as long as we deal with global supersymmetry. However, supergravity corrections include a term $M_{\mathrm{Pl}}^{-1} \psi_{m}^{(2)} S^{(2) m}$, where $\psi_{m}^{(2)}$ is the second gravitino and $S^{(2) m}$ is the Noether current associated with the second supersymmetry.

\footnotetext{
${ }^{5}$ This reasoning is valid before integrating out the auxiliary fields $D, F$ of the bulk vector multiplet. After integrating out $D, F$, the Langrangian contains direct couplings between the two boundary sectors (this has been found in a similar context in [32]). For our toy model, we implicitly assume that the two boundary sectors are seperated by some extra dimension such that direct couplings between the two boundaries are absent even after integrating out the auxiliary fields (see, e.g., [11).
} 
From the definition of the supercurrent,

$$
\delta_{\eta, \text { local }}^{\star} \mathcal{L}_{\text {global }}=S^{(2) m} \partial_{m} \eta+\bar{S}^{(2) m} \partial_{m} \bar{\eta}
$$

it is clear that $S^{(2) m}$ has no well-defined R-charge if $\eta$ has no well-defined R-charge.

Interestingly, there is still an unbroken $\mathbb{Z}_{4}$-symmetry that protects the fermions from acquiring masses. Under this $\mathbb{Z}_{4}$, all fields except $\Lambda_{g}$ and $\Lambda_{g}^{\prime}$ have charges as in table $\mathbb{1}$. The charges of the goldstino superfields are flipped, i.e., $\Lambda_{g}$ has charge -1 and $\Lambda_{g}^{\prime}$ has charge 1. This implies that the Lagrangian (6.4) is $\mathbb{Z}_{4}$-invariant. It is easy to see that the supercurrents have well-defined $\mathbb{Z}_{4}$-charges $\left(S^{(1) m}:-1, S^{(2) m}: 1\right)$ and that all interactions, including the coupling to supergravity, are $\mathbb{Z}_{4}$-invariant if we assign charge 1 to $\psi_{m}^{(1)}$ and charge -1 to $\psi_{m}^{(2)}$.

Naively, one would expect that the second gravitino acquires a mass by eating the first goldstino. Indeed, the coupling of the the second gravitino to the second supercurrent, $M_{\mathrm{Pl}}^{-1} \psi_{m}^{(2)} S^{(2) m}$, contains a term ${ }^{\mathbb{9}}\left(M_{\mathrm{Pl}} \kappa\right)^{-1} \psi_{m}^{(2)} \sigma^{m} \bar{\lambda}_{g}$. But it is not possible to eliminate the goldstino degrees of freedom by shifting the gravitino field appropriately (see, e.g., [34]), $\psi_{m}^{(2)^{\prime}}=\psi_{m}^{(2)}+\left(2 \partial_{m} \lambda_{g}+i m \sigma_{m} \bar{\lambda}_{g}\right) / \sqrt{6} m$, with $m=\left(M_{\mathrm{Pl}} \kappa\right)^{-1}$. The reason is that there is no mass term for $\lambda_{g}$. The goldstino remains massless even when coupled to supergravity. Another way to see this is by considering the sector $\mathcal{L}_{1}+\mathcal{L}_{\text {bulk }}$, which has unbroken $\mathcal{N}=1$ supersymmetry. The goldstino must remain massless because it is the superpartner of the massless $U(1)$ gauge boson. This has the surprising consequence that the second gravitino, too, remains massless. Similarly, one finds that the first gravitino does not acquire a mass. Higher loop diagrams involving fields from all three sectors invalidate this argument and most probably will generate gravitino masses. Since the goldstinos are neutral under the bulk gauge symmetry, possible diagrams contributing to goldstino masses only arise at three loops. The first contribution to the vacuum energy appears at the same loop order. This leads to a vacuum expectation value for one of the auxiliary fields of the gravity multiplet, which cancels the vacuum energy term. This vacuum expectation value breaks the abovementioned $\mathbb{Z}_{4}$-symmetry and generates gravitino masses.

\section{Conclusions and outlook}

We have developed a formalism to study effective field theory descriptions of pseudo-supersymmetry. This is a supersymmetry breaking mechanism that naturally arises in many open string models. Typically, such models contain a bulk sector with extended supersymmetry and boundary sectors that break different fractions of the bulk supersymmetry. But the complete supersymmetry is still non-linearly realized. We have shown how to consistently couple $\mathcal{N}=2$ supersymmetric multiplets in the bulk to $\mathcal{N}=1$ matter on the boundaries. For a pseudo-supersymmetry toy model containing an $\mathcal{N}=2$ vector in the bulk, we have computed explicitly the goldstino couplings. One interesting result is that gravitino masses arise only at three loops.

\footnotetext{
${ }^{6} \kappa^{-1 / 2}$ is the supersymmetry breaking scale; this is not related to the gravitational coupling, which is set by $M_{\mathrm{Pl}}$.

${ }^{7}$ Note that a similar result was found for a ten-dimensional non-supersymmetric type I string model [12].
} 
There are several interesting directions for further research. First, it is a straightforward exercise to apply the formalism of this article to concrete D-brane models and to compute explicitly the scalar masses arising at two loops.

Second, it is important to understand the coupling to supergravity in more detail. This was only sketched very roughly in the previous section. Most probably, one would gain new insights by generalizing the partial supergravity breaking of [34 to local pseudo-supersymmetry [10]. It is also interesting to analyze the mechanism that breaks the $\mathbb{Z}_{4}$-symmetry and generates gravitino masses.

Finally, the coupling of bulk hyper multiplets to boundary gauge multiplets is not discussed in this paper. It would certainly be very interesting to determine these couplings. For a hyper multiplet $\boldsymbol{\Phi}=\left(\Phi_{1}, \Phi_{2}\right)$, where $\Phi_{1}, \Phi_{2}$ are chiral multiplets, it is easy to construct a composite superfield $\hat{\Phi}_{1}$ that transforms linearly under the first and non-linearly under the second supersymmetry. However, $\hat{\Phi}_{1}$ is not a chiral superfield and therefore cannot be consistently coupled to the gauge-kinetic terms of the boundary sector. It is well-known [35] that in linear $\mathcal{N}=2$ supersymmetry, it is not possible to couple hyper multiplets to the gauge kinetic terms of vector multiplets. The results of the previous sections seem to suggest that it is not even possible to couple components of hyper multiplets to gauge kinetic terms if the second supersymmetry is non-linearly realized. This leads to a puzzle. In $\mathcal{N}=2$ supersymmetric type II string vacua, the dilaton resides in a hyper multiplet. If there are D-branes, then the dilaton couples to the gauge-kinetic terms on their world-volume. It is not clear how this coupling can be rendered non-linearly $\mathcal{N}=2$ invariant.

\section{Acknowledgements}

It is a pleasure to thank Michael Peskin for many stimulating discussions and for proofreading the manuscript. My interest in pseudo-supersymmetry was strongly influenced by discussions with Cliff Burgess, Elise Filotas and Fernando Quevedo. I have also benefited from discussions with Ignatios Antoniadis, Shamit Kachru and Albion Lawrence. Special thanks go to my wife for her support and encouragement. This research is funded by the Deutsche Forschungsgemeinschaft.

\section{A Notation and useful formulae}

\section{A.1 Spinors}

We use the metric $\eta_{m n}=\operatorname{diag}(-1,1,1,1)$ and the spinor conventions of Wess and Bagger 20]. All spinors appearing in this article are 2-component Weyl spinors. A dot on an spinor index indicates that the corresponding field transforms according to the conjugate spinor representation of the Lorentz group. Indices are raised and lowered with the help of the antisymmetric tensors $\epsilon^{\alpha \beta}, \epsilon_{\alpha \beta}\left(\epsilon^{12}=\epsilon_{21}=1\right)$ :

$$
\psi^{\alpha}=\epsilon^{\alpha \beta} \psi_{\beta}, \quad \psi_{\alpha}=\epsilon_{\alpha \beta} \psi^{\beta},
$$




$$
\begin{aligned}
& \bar{\psi}^{\dot{\alpha}}=\epsilon^{\dot{\alpha} \dot{\beta}} \bar{\psi}_{\dot{\beta}}, \quad \bar{\psi}_{\dot{\alpha}}=\epsilon_{\dot{\alpha} \dot{\beta}} \bar{\psi}^{\dot{\beta}}, \\
& \bar{\sigma}^{m \dot{\alpha} \alpha}=\epsilon^{\dot{\alpha} \dot{\beta}} \epsilon^{\alpha \beta} \sigma_{\beta \dot{\beta}}^{m} .
\end{aligned}
$$

When spinor indices are suppressed, they are contracted as follows:

$$
\psi \chi=\psi^{\alpha} \chi_{\alpha}, \quad \bar{\psi} \bar{\chi}=\bar{\psi}_{\dot{\alpha}} \bar{\chi}^{\dot{\alpha}}, \quad \psi \sigma^{m} \bar{\chi}=\psi^{\alpha} \sigma_{\alpha \dot{\alpha}}^{m} \bar{\chi}^{\dot{\alpha}} .
$$

Complex conjugation acts as

$$
\left(\psi_{\alpha}\right)^{\dagger}=\bar{\psi}_{\dot{\alpha}}, \quad(\psi \chi)^{\dagger}=\bar{\chi} \bar{\psi}, \quad\left(\psi \sigma^{m} \bar{\chi}\right)^{\dagger}=\chi \sigma^{m} \bar{\psi} .
$$

Some useful identities for the $\sigma$-matrices are

$$
\begin{aligned}
\left(\sigma^{m n}\right)_{\alpha}^{\beta} & \equiv \frac{1}{4}\left(\sigma^{m} \bar{\sigma}^{n}-\sigma^{n} \bar{\sigma}^{m}\right)_{\alpha}^{\beta}, \\
\left(\sigma^{m} \bar{\sigma}^{n}+\sigma^{n} \bar{\sigma}^{m}\right)_{\alpha}^{\beta} & =-2 \eta^{m n} \delta_{\alpha}^{\beta}, \\
\sigma_{\alpha \dot{\alpha}}^{m} \bar{\sigma}_{m}^{\dot{\beta} \beta} & =-2 \delta_{\alpha}^{\beta} \delta_{\dot{\alpha}}^{\dot{\beta}}, \\
\frac{i}{2} \epsilon^{m n p q} \sigma_{p q} & =\sigma^{m n} .
\end{aligned}
$$

Some useful Weyl spinor identities are

$$
\begin{aligned}
\theta^{\alpha} \theta^{\beta} & =-\frac{1}{2} \epsilon^{\alpha \beta} \theta \theta, \quad \bar{\theta}_{\dot{\alpha}} \bar{\theta}_{\dot{\beta}}=-\frac{1}{2} \epsilon_{\dot{\alpha} \dot{\beta}} \bar{\theta} \bar{\theta} \\
\theta_{\alpha} \theta_{\beta} & =\frac{1}{2} \epsilon_{\alpha \beta} \theta \theta, \quad \bar{\theta}^{\dot{\alpha}} \bar{\theta}^{\dot{\beta}}=\frac{1}{2} \epsilon^{\dot{\alpha} \dot{\beta}} \bar{\theta} \bar{\theta} \\
\psi \chi & =\chi \psi \\
\psi \sigma^{m} \bar{\chi} & =-\bar{\chi} \bar{\sigma}^{m} \psi, \\
\psi \sigma^{m n} \chi & =\chi \sigma^{n m} \psi \\
(\theta \psi)(\chi \eta) & =-\frac{1}{2}\left[(\theta \eta)(\chi \psi)+\left(\theta \sigma^{m n} \eta\right)\left(\chi \sigma_{m n} \psi\right)\right] \\
(\theta \psi)(\bar{\chi} \bar{\eta}) & =\frac{1}{2}\left(\theta \sigma^{m} \bar{\eta}\right)\left(\bar{\chi} \bar{\sigma}_{m} \psi\right) .
\end{aligned}
$$

\section{A.2 Supersymmetry}

The $\mathcal{N}=2$ supersymmetry algebra without central charges is

$$
\left\{Q_{\alpha}, \bar{Q}_{\dot{\beta}}\right\}=\left\{S_{\alpha}, \bar{S}_{\dot{\beta}}\right\}=2 \sigma_{\alpha \dot{\beta}}^{m} P_{m}
$$

with all other anticommutators vanishing. When acting on fields, $P_{m}=-i \partial_{m}$. The $\mathcal{N}=1$ covariant derivatives $D_{\alpha}, \bar{D}_{\dot{\alpha}}$ are represented by differential operators on superspace,

$$
D_{\alpha}=\frac{\partial}{\partial \theta^{\alpha}}+i\left(\sigma^{m} \bar{\theta}\right)_{\alpha} \partial_{m}, \quad \bar{D}_{\dot{\alpha}}=-\frac{\partial}{\partial \bar{\theta}^{\dot{\alpha}}}-i\left(\theta \sigma^{m}\right)_{\dot{\alpha}} \partial_{m}
$$


They satisfy the anticommutation relations

$$
\left\{D_{\alpha}, D_{\beta}\right\}=0, \quad\left\{D_{\alpha}, \bar{D}_{\dot{\beta}}\right\}=-2 i \sigma_{\alpha \dot{\beta}}^{m} \partial_{m}
$$

From (A.16), we find

$$
\begin{aligned}
D^{2} & \equiv D^{\alpha} D_{\alpha}=-\frac{\partial}{\partial \theta} \frac{\partial}{\partial \theta}-2 i \bar{\theta} \bar{\sigma}^{m} \frac{\partial}{\partial \theta} \partial_{m}-\bar{\theta} \bar{\theta} \square \\
\bar{D}^{2} & \equiv \bar{D}_{\dot{\alpha}} D^{\dot{\alpha}}=-\frac{\partial}{\partial \bar{\theta}} \frac{\partial}{\partial \bar{\theta}}-2 i \theta \sigma^{m} \frac{\partial}{\partial \bar{\theta}} \partial_{m}-\theta \theta \square
\end{aligned}
$$

Integration of Grassmann variables and derivation with respect to them is essentially equivalent. Normalizing the Grassmann integral measure such that $\int d^{2} \theta \theta \theta=1$, one has

$$
\int d^{2} \theta=-\frac{1}{4} D^{2}+\text { total deriv., } \int d^{2} \bar{\theta}=-\frac{1}{4} \bar{D}^{2}+\text { total deriv. }
$$

A chiral superfield $\Phi(x, \theta, \bar{\theta})$ is defined by the condition $\bar{D}_{\dot{\alpha}} \Phi=0$. Its complex conjugate is an antichiral superfield, i.e., $D_{\alpha} \Phi^{\dagger}=0$. For a chiral superfield

$$
\Phi=\phi+i \theta \sigma^{m} \bar{\theta} \partial_{m} \phi+\frac{1}{4} \theta \theta \bar{\theta} \bar{\theta} \square \phi+\sqrt{2} \theta \psi-\frac{i}{\sqrt{2}} \theta \theta \partial_{m} \psi \sigma^{m} \bar{\theta}+\theta \theta F
$$

one has

$$
-\frac{1}{4} D^{2} \Phi=F+i \sqrt{2} \bar{\theta} \bar{\sigma}^{m} \partial_{m} \psi-i \theta \sigma^{m} \bar{\theta} \partial_{m} F+\bar{\theta} \bar{\theta} \square \phi+\frac{1}{\sqrt{2}} \bar{\theta} \bar{\theta} \theta \square \psi+\frac{1}{4} \theta \theta \bar{\theta} \bar{\theta} \square F,
$$

which is an antichiral superfield, since $D_{\alpha} D_{\beta} D_{\gamma} \equiv 0$. For the $\mathcal{N}=1$ chiral field strength superfield

$$
\begin{aligned}
W_{\alpha}= & -i \lambda_{\alpha}+\theta_{\alpha} D-i\left(\sigma^{m n} \theta\right)_{\alpha} F_{m n}+\theta \sigma^{m} \bar{\theta} \partial_{m} \lambda_{\alpha}+\theta \theta\left(\sigma^{m} \partial_{m} \bar{\lambda}\right)_{\alpha} \\
& -\frac{1}{2} \theta \theta\left(\sigma^{m} \bar{\theta}\right)_{\alpha}\left(i \partial_{m} D-\partial^{n} F_{m n}\right)-\frac{i}{4} \theta \theta \bar{\theta} \bar{\theta} \square \lambda_{\alpha}
\end{aligned}
$$

this yields

$$
-\frac{1}{4} D^{2} W \eta=-i \eta \sigma^{m} \partial_{m} \bar{W}
$$

The $\mathcal{N}=2$ covariant derivatives introduced on page 13 are given by 9

$$
\begin{aligned}
\mathcal{D}_{\alpha} & =D_{\alpha}+i \kappa^{2}\left(D_{\alpha} \Lambda_{g} \sigma^{m} \bar{\Lambda}_{g}+D_{\alpha} \bar{\Lambda}_{g} \bar{\sigma}^{m} \Lambda_{g}\right) D_{m} \\
\overline{\mathcal{D}}_{\dot{\alpha}} & =\bar{D}_{\dot{\alpha}}+i \kappa^{2}\left(\bar{D}_{\dot{\alpha}} \Lambda_{g} \sigma^{m} \bar{\Lambda}_{g}+\bar{D}_{\dot{\alpha}} \bar{\Lambda}_{g} \bar{\sigma}^{m} \Lambda_{g}\right) D_{m} \\
D_{m} & =\left(\omega^{-1}\right)_{m}^{n} \partial_{n}
\end{aligned}
$$

where $\omega_{m}^{n}$ is a generalization of $(2.3)$ to the case of partially broken $\mathcal{N}=2$ supersymmetry,

$$
\omega_{m}^{n}=\delta_{m}^{n}-i \kappa^{2}\left(\partial_{m} \Lambda_{g} \sigma^{n} \bar{\Lambda}_{g}+\partial_{m} \bar{\Lambda}_{g} \bar{\sigma}^{n} \Lambda_{g}\right)
$$


These are derivatives with respect to the first (linearly realized) supersymmetry but they are covariant with respect to the second (non-linearly realized) supersymmetry in the sense that they satisfy (4.20). They generate the following algebra [9]

$$
\begin{aligned}
& \left\{\mathcal{D}_{\alpha}, \mathcal{D}_{\beta}\right\}=2 i \kappa^{2}\left(\mathcal{D}_{\alpha}\left(\Lambda_{g} \sigma^{m}\right)_{\dot{\gamma}} \mathcal{D}_{\beta} \bar{\Lambda}_{g}^{\dot{\gamma}}+\mathcal{D}_{\beta}\left(\Lambda_{g} \sigma^{m}\right)_{\dot{\gamma}} \mathcal{D}_{\alpha} \bar{\Lambda}_{g}^{\dot{\gamma}}\right) D_{m}, \\
& \left\{\mathcal{D}_{\alpha}, \overline{\mathcal{D}}_{\dot{\beta}}\right\}=-2 i \sigma_{\alpha \dot{\beta}}^{m} D_{m}+2 i \kappa^{2}\left(\mathcal{D}_{\alpha}\left(\Lambda_{g} \sigma^{m}\right)_{\dot{\gamma}} \overline{\mathcal{D}}_{\dot{\beta}} \bar{\Lambda}_{g}^{\dot{\gamma}}+\overline{\mathcal{D}}_{\dot{\beta}}\left(\Lambda_{g} \sigma^{m}\right)_{\dot{\gamma}} \mathcal{D}_{\alpha} \bar{\Lambda} \dot{\gamma}\right) D_{m}, \\
& {\left[\mathcal{D}_{\alpha}, D_{m}\right]=2 i \kappa^{2}\left(\mathcal{D}_{\alpha}\left(\Lambda_{g} \sigma^{n}\right)_{\dot{\gamma}} D_{m} \bar{\Lambda}_{g}^{\dot{\gamma}}+D_{m}\left(\Lambda_{g} \sigma^{n}\right)_{\dot{\gamma}} \mathcal{D}_{\alpha} \bar{\Lambda}_{g}^{\dot{\gamma}}\right) D_{n} .}
\end{aligned}
$$

Due to the fact that $\tilde{\Lambda}_{g}$ is an $\mathcal{N}=1$ chiral superfield, which is equivalent to the condition

$$
\overline{\mathcal{D}}_{\dot{\alpha}} \Lambda_{g}=0
$$

the algebra (A.26) simplifies to

$$
\begin{aligned}
& \left\{\mathcal{D}_{\alpha}, \mathcal{D}_{\beta}\right\}=0 \\
& \left\{\mathcal{D}_{\alpha}, \overline{\mathcal{D}}_{\dot{\beta}}\right\}=-2 i \sigma_{\alpha \dot{\beta}}^{m} D_{m}+2 i \kappa^{2} \mathcal{D}_{\alpha}\left(\Lambda_{g} \sigma^{m}\right)_{\dot{\gamma}} \overline{\mathcal{D}}_{\dot{\beta}} \bar{\Lambda}_{g}^{\dot{\gamma}} D_{m} \\
& {\left[\mathcal{D}_{\alpha}, D_{m}\right]=2 i \kappa^{2} \mathcal{D}_{\alpha}\left(\Lambda_{g} \sigma^{n}\right)_{\dot{\gamma}} D_{m} \bar{\Lambda}_{g}^{\dot{\gamma}} D_{n}}
\end{aligned}
$$

\section{References}

[1] J. Polchinski, "Dirichlet Branes And Ramond-Ramond Charges", Phys. Rev. Lett. 75 (1995) 4724, hep-th/9510017.

[2] I. Antoniadis, E. Dudas, A. Sagnotti, "Brane Supersymmetry Breaking", Phys. Lett. 464B (1999) 38, hep-th/9908023.

[3] G. Aldazabal, A. M. Uranga, "Tachyon Free Nonsupersymmetric Type IIB Orientifolds Via Brane - Anti-Brane Systems", JHEP 9910 (1999) 024, hep-th/9908072.

[4] G. Aldazabal, L. E. Ibáñez, F. Quevedo, "Standard - Like Models With Broken Supersymmetry From Type I String Vacua", JHEP 0001 (2000) 031, hep-th/9909172.

[5] C. Angelantonj, I. Antoniadis, G. D’Appollonio, E. Dudas, A. Sagnotti, "Type I vacua with brane supersymmetry breaking", Nucl. Phys. B572 (2000) 36, hep-th/9911081.

[6] R. Blumenhagen, L. Görlich, B. Körs, D. Lüst, "Noncommutative Compactifications Of Type I Strings On Tori With Magnetic Background Flux, JHEP 0010 (2000) 006, hep-th/0007024; R. Blumenhagen, B. Körs, D. Lüst, T. Ott, "The Standard Model From Stable Intersecting Brane World Orbifolds", Nucl. Phys. B616 (2001) 3, hepth/0107138.

[7] D. Cremades, L. E. Ibáñez, F. Marchesano, "SUSY Quivers, Intersecting Branes and the Modest Hierarchy Problem", hep-th/0201205; "Intersecting Brane Models of Particle Physics and the Higgs Mechanism", hep-th/0203160. 
[8] J. Polchinski, "String Theory", Ch. 13.2, Cambridge University Press (1998).

[9] J. Bagger, A. Galperin, "New Goldstone multiplet for partially broken supersymmetry", Phys. Rev. D55 (1997) 1091, hep-th/9608177.

[10] C. Burgess, E. Filotas, M. Klein, F. Quevedo, work in progress.

[11] E. A. Mirabelli, M. E. Peskin, "Transmission Of Supersymmetry Breaking From A Four-Dimensional Boundary", Phys. Rev. D58 (1998) 065002, hep-th/9712214.

[12] E. Dudas, J. Mourad, "Consistent gravitino couplings in non-supersymmetric strings", Phys. Lett. 514B (2001) 173, hep-th/0012071.

[13] G. Pradisi, F. Riccioni, "Geometric Couplings and Brane Supersymmetry Breaking, Nucl. Phys. B615 (2001) 33, hep-th/0107090.

[14] I. Antoniadis, K. Benakli, A. Laugier, "D-brane Models with Non-Linear Supersymmetry", hep-th/0111209.

[15] D. V. Volkov, V. P. Akulov, "Possible Universal Neutrino Interaction", JETP Lett. 16 (1972) 438; "Is The Neutrino A Goldstone Particle?", Phys. Lett. 46B (1973) 109.

[16] V. P. Akulov, D. V. Volkov, "Goldstone Fields With Spin 1/2", Theor. Math. Phys. 18 (1974) 28.

[17] A. I. Pashnev, "Nonlinear Realization For Symmetry Group With Spinor Parameters", Theor. Math. Phys. 20 (1974) 725.

[18] D. V. Volkov, V. A. Soroka, "Higgs Effect For Goldstone Particles With Spin 1/2" JETP Lett. 18 (1973) 312.

[19] J. Wess, B. Zumino, "Supergauge Transformations In Four-Dimensions", Nucl. Phys. B70 (1974) 39.

[20] J. Wess, J. Bagger, "Supersymmetry and Supergravity", 2nd ed., Princeton University Press (1992).

[21] E. A. Ivanov, A. A. Kapustnikov, "General relationship between linear and nonlinear realisations of supersymmetry", J. Phys. A11 (1978) 2375.

[22] T. E. Clark, S. T. Love, "Goldstino Couplings to Matter", Phys. Rev. D54 (1996) 5723, hep-ph/9608243.

[23] T. E. Clark, T. Lee, S. T. Love, G. H. Wu, "On the Interactions of Light Gravitinos", Phys. Rev. D57 (1998) 5912, hep-ph/9712353.

[24] B. Zumino, "Fermi-Bose Supersymmetry (Supergauge Symmetry In Four-Dimensions)", Proc. of 17th Int. Conf. on High Energy Physics, Imperial College, London, England, Jul 1-10, 1974, J. R. Smith, ed., Rutherford Lab. (1974) I-254. 
[25] S. Samuel, J. Wess, "A superfield formulation of the nonlinear realization of supersymmetry and its coupling to supergravity", Nucl. Phys. B221 (1983) 153.

[26] J. Bagger, A. Galperin, "Matter couplings in partially broken extended supersymmetry", Phys. Lett. 336B (1994) 25, hep-th/9406217.

[27] J. Bagger, A. Galperin, "The tensor Goldstone multiplet for partially broken supersymmetry", Phys. Lett. 412B (1997) 296, hep-th/9707061.

[28] J. Bagger, J. Wess, "Partial breaking of extended supersymmetry", Phys. Lett. 138B (1984) 105.

[29] M. Born, L. Infeld, "Foundations of the new field theory", Proc. Roy. Soc. Lond. A144 (1934) 425; R. G. Leigh, "Dirac-Born-Infeld action from Dirichlet sigma model", Mod. Phys. Lett. A4 (1989) 2767.

[30] P. West, "Introduction to supersymmetry and supergravity", World Scientific (1986).

[31] G. Aldazabal, L. E. Ibáñez, F. Quevedo, A. M. Uranga, "D-Branes at Singularities: A Bottom-Up Approach to the String Embedding of the Standard Model", JHEP 0008 (2000) 002, hep-th/0005067.

[32] A. Anisimov, M. Dine, M. Graesser, S. Thomas, "Brane World Susy Breaking From String / M Theory", hep-th/0201256.

[33] N. Arkani-Hamed, A. G. Cohen, H. Georgi, "Twisted Supersymmetry And The Topology Of Theory Space", hep-th/0109082.

[34] R. Altendorfer, J. Bagger, "Dual Supersymmetry Algebras from Partial Supersymmetry Breaking", Phys. Lett. 460B (1999) 127, hep-th/9904213.

[35] B. de Wit, P. G. Lauwers, A. Van Proeyen, "Lagrangians Of N=2 Supergravity - Matter Systems", Nucl. Phys. B255 (1985) 569. 